\title{
Computations of Green's Function and Its Fourier Coefficients on Fuchsian Groups
}

\author{
Helen Avelin
}

\section{CONTENTS}

1. Introduction

2. Preliminaries

3. Computations of $\boldsymbol{F}_{n}(z ; s)$

4. Computations of $G_{s}(z ; w)$

5. Computations of Bessel Functions of Imaginary Order

6. Numerics

7. Results

Acknowledgments

References

2000 AMS Subject Classification: Primary 11F03; Secondary 11-04, 11Y16, 33F05, 34B27

Keywords: Green's function, resolvent kernel, Rankin-Selberg bound, computational number theory, Fourier coefficients, algorithms, spectral theory, $K$-Bessel function, $I$-Bessel function.
We develop algorithms for computations of Green's function and its Fourier coefficients, $F_{n}(z ; s)$, on Fuchsian groups with one cusp. An analogue of a Rankin-Selberg bound for $F_{n}(z ; s)$ is presented.

\section{INTRODUCTION}

The possibility of proving the Riemann hypothesis by finding a self-adjoint operator on a Hilbert space whose eigenvalues are precisely the imaginary parts of the nontrivial zeros of the Riemann zeta function was suggested independently by Pólya and Hilbert around 1910. Apparently, neither of them had any specific space or operator in mind; see, for example, [Conrey 03, Odlyzko 09].

In 1977, an experiment conducted by Hartmut Haas seemed to produce numerical evidence that the HilbertPólya operator had been discovered. Indeed, the first few zeros of the Riemann zeta function seemed to appear in Haas's attempt to compute eigenvalues of the Laplacian on $\operatorname{PSL}(2, \mathbb{Z}) \backslash \mathcal{H}$, where $\mathcal{H}$ is the Poincaré upper halfplane.

However, the true circumstances were revealed when Dennis Hejhal investigated the matter more closely [Hejhal 81]. He found that Haas had forgotten to take necessary precautions to rule out the possibility of singularities. The eigenfunctions corresponding to the remarkable eigenvalues all had logarithmic singularities at $1 / 2+i \sqrt{3} / 2$.

These events triggered Hejhal's interest in cusp forms with logarithmic singularities, which he named pseudo cusp forms. Elaborating on Haas's somewhat accidental method of computing them, Hejhal was able find many more eigenvalues of pseudo cusp forms. However, several open questions still remain to be explored; see [Hejhal 81, Hejhal 92]. 
A pseudo cusp form is a special case of Green's function. In the present paper we develop a method for numerical computation of Green's function (i.e., the resolvent kernel of the Laplacian) on Fuchsian groups. Our method, which builds on Hejhal's algorithm for cusp forms, is designed for eigenfunctions with logarithmic singularities. The primary computational challenges are that multiple layers of singularities have to be dealt with, and that the Fourier coefficients increase very rapidly in magnitude.

Green's function is important in the spectral theory on hyperbolic manifolds, e.g., in the theory of the Selberg trace formula; cf. [Hejhal 83, Niebur 73, Fay 77]. Moreover, both Green's function and its Fourier coefficients, $F_{n}(z ; s)$, are important objects in the study of the singular theta correspondence; see, for example, [Borcherds 98, Brunier 02, Barnard 03].

It is well known that the Fourier coefficients themselves can be written as Fourier expansions and that in some sense, $F_{n}(z ; s)$ is a kind of generalized Eisenstein series. In Section 3 we show how the algorithm for computing Eisenstein series described in [Avelin 07] may be adapted to the Fourier coefficients of Green's function. The algorithm for $G_{s}(z ; w)$ is described in Section 4 .

Eigenfunctions of the Laplacian usually involve Bessel functions. Here it is the modified Bessel function of the second kind, $K_{\nu}(x)$, and the modified Bessel function of the first kind, $I_{\nu}(x)$, that appear, and we compute them for $\nu=i R, R \in \mathbb{R}$, with power series expansions and asymptotic expansions. In Section 5 we give a detailed description of our techniques for these computations.

After presenting the main ideas of our algorithms, we discuss some specifics of the computations in Section 6, and finally, we devote Section 7 to numerical results and tests of the algorithms for $\operatorname{Re} s=1 / 2$. In [Avelin 10], we use the algorithms for various statistical tests of $G_{s}(z ; w)$ and $F_{n}(z ; s)$.

We will begin by providing the necessary theoretical background.

\section{PRELIMINARIES}

Let $\Gamma$ be a cofinite Fuchsian group acting on the Poincaré upper half-plane $\mathcal{H}=\{x+i y: y>0\}$ equipped with the hyperbolic metric $d s^{2}=y^{-2}\left(d x^{2}+d y^{2}\right)$ and area measure $d \mu(x+i y)=y^{-2} d x d y$. The functions that we study are eigenfunctions of the Laplace-Beltrami operator on $\Gamma \backslash \mathcal{H}$ :

$$
\Delta=y^{2}\left(\frac{\partial^{2}}{\partial x^{2}}+\frac{\partial^{2}}{\partial y^{2}}\right) \text {. }
$$

Let $K_{\nu}(x)$ and $I_{\nu}(x)$ denote the $K$-Bessel and the $I$-Bessel functions respectively. These will play an important role in the present paper, so it will be convenient to have some estimates at hand. As $x \rightarrow \infty$ we have the following asymptotic series expansions:

$$
\begin{aligned}
K_{\nu}(x) & \sim \frac{\sqrt{\pi} e^{-x}}{\sqrt{2 x}} \sum_{m=0}^{\infty} \frac{A_{m}(\nu)}{x^{m}}, \\
I_{\nu}(x) & \sim \frac{e^{x}}{\sqrt{2 \pi x}} \sum_{m=0}^{\infty} \frac{(-1)^{m} A_{m}(\nu)}{x^{m}},
\end{aligned}
$$

with

$$
\begin{aligned}
A_{0}(\nu) & =1, \\
A_{m}(\nu) & =\frac{\left(4 \nu^{2}-1\right)\left(4 \nu^{2}-3^{2}\right) \cdots\left(4 \nu^{2}-(2 m-1)^{2}\right)}{m ! 8^{m}} ;
\end{aligned}
$$

cf. [Abramowitz and Stegun 64, pp. 377-378] or [Olver 74, pp. 132, 269].

Throughout this paper we assume that $\Gamma$ has only one cusp, situated at $i \infty$, and is normalized so that $\Gamma_{\infty}=[S]$, where $S z=z+1$ for $z \in \mathcal{H}$. Let $\mathcal{F}$ denote a fundamental geodesic polygon of $\Gamma$ such that for $y$ larger than some $B_{0}>1, \mathcal{F}$ consists of only the vertical strip $\{x+i y \in$ $\left.\mathcal{H}:-1 / 2 \leq x \leq 1 / 2, y>B_{0}\right\}$.

We define the point-pair invariant $k_{s}(z ; w)$, for $z, w \in$ $\mathcal{H}, z \neq w, s \in \mathbb{C}$, by

$$
\begin{aligned}
k_{s}(z ; w)= & -\frac{\Gamma(s)^{2}}{4 \pi \Gamma(2 s)}\left(1-\left|\frac{z-w}{z-\bar{w}}\right|^{2}\right) \\
& \times F\left(s, s ; 2 s ; 1-\left|\frac{z-w}{z-\bar{w}}\right|^{2}\right),
\end{aligned}
$$

where $F(a, b ; c ; d)$ is the hypergeometric function (cf. [Hejhal 83, pp. 31, 602ff.] or [Abramowitz and Stegun 64, p. 556]). Note that $k_{s}(z ; w)$ is a meromorphic function of $s \in \mathbb{C}$ with no poles outside the set $s \in\{0,-1,-2,-3, \ldots\}$. By [Hejhal 83, Section 6.4, especially (4.1), (4.2)] we know that for $s \in$ $\mathbb{C} \backslash\{0,-1,-2,-3, \ldots\}, k_{s}(z ; w)$ satisfies the differential equation $\Delta_{z} f+s(1-s) f=0$ when $z \neq w$, and that $k_{s}(z ; w)=\frac{1}{2 \pi} \ln |z-w|+O(1)$ for $z$ near $w$.

The automorphic Green's function is defined by adding over group elements $T \in \Gamma$ :

$$
G_{s}(z ; w)=\sum_{T \in \Gamma} k_{s}(T z ; w) .
$$

This sum is convergent whenever $z \neq w \bmod \Gamma$ and $\operatorname{Re} s>1$. One proves that $G_{s}(z ; w)=G_{s}(w ; z)$; that $G_{s}(z ; w)$ is automorphic in both variables, i.e., 
$G_{s}(T z ; U w)=G_{s}(z ; w)$ when $T, U \in \Gamma ;$ and that $G_{s}(z ; w)$ satisfies $\Delta_{z} f+s(1-s) f=0$ when $z \neq w$ $\bmod \Gamma$; cf. [Hejhal 83, p. 33, Proposition 6.5].

The function $G_{s}(z ; w)$ has a meromorphic continuation to all of $s \in \mathbb{C}$, and for $s$ not a pole the following Fourier series representation holds for $z=x+i y$ with $y>\operatorname{Im} T w$ for all $T \in \Gamma$ (cf. [Hejhal 83, pp. 39-42, 250] and Lemma 2.1 (a) below):

$$
\begin{aligned}
G_{s}(z ; w)= & \frac{E(w ; s) y^{1-s}}{1-2 s} \\
& -\sum_{n \neq 0} F_{-n}(w ; s) y^{1 / 2} K_{s-\frac{1}{2}}(2 \pi|n| y) e^{2 \pi i n x}
\end{aligned}
$$

The Fourier coefficients of (2-4) are given by (for $n \neq 0$ and Re $s>1$ ), cf. [Hejhal 83, p. 41 (6.7)],

$$
\begin{aligned}
& F_{n}(z ; s) \\
& \quad=\sum_{W_{0} \in \Gamma_{\infty} \backslash \Gamma}\left(\operatorname{Im} W_{0} z\right)^{1 / 2} I_{s-\frac{1}{2}}\left(2 \pi|n| \operatorname{Im} W_{0} z\right) e^{2 \pi i n \operatorname{Re} W_{0} z},
\end{aligned}
$$

and $F_{0}(z ; s)=E(z ; s)$, i.e., the Eisenstein series. Each $F_{n}(z ; s)$ has a Fourier series

$$
\begin{aligned}
F_{n}(z ; s)= & \begin{cases}y^{1 / 2} I_{s-\frac{1}{2}}(2 \pi|n| y) e^{2 \pi i n x}, & n \neq 0, \\
y^{s}, & n=0,\end{cases} \\
& +\varphi_{0}^{n}(s) \frac{y^{1-s}}{2 s-1} \\
& +\sum_{m \neq 0} \varphi_{m}^{n}(s) y^{1 / 2} K_{s-\frac{1}{2}}(2 \pi|m| y) e^{2 \pi i m x},
\end{aligned}
$$

valid for $s \in \mathbb{C} \backslash\left\{\frac{1}{2}\right\}, s$ not a pole; cf. [Hejhal 83, pp. 56ff., 254ff.].

In the next lemma we gather some symmetry relations for $G_{s}(z ; w), F_{n}(z ; s)$, and $\varphi_{m}^{n}(s)$. Let $\mathcal{R}$ be the reflection $\mathcal{R} z=-\bar{z}$.

Lemma 2.1. The following relations hold for $s \in \mathbb{C}$ and $z, w \in \mathcal{H}$ when $s$ is not a pole and $w \notin \Gamma z$.

(a) $\overline{G_{\bar{s}}(z ; w)}=G_{s}(z ; w), \overline{F_{n}(z ; \bar{s})}=F_{-n}(z ; s), n \in \mathbb{Z}$.

(b) $F_{n}(z ; s)-F_{n}(z ; 1-s)=\varphi_{-n}^{0}(s) E(z ; 1-s) /(2 s-1)$, $n \neq 0$.

Assume that $\Gamma \mathcal{R}=\mathcal{R} \Gamma$. Then

(c) $G_{s}(\mathcal{R} z ; \mathcal{R} w)=G_{s}(z ; w)$.

(d) If $\mathcal{R} w=T w$ for some $T \in \Gamma$, then $G_{s}(x+i y ; w)$ is even in $x$

(e) $F_{n}(z ; s)=F_{-n}(\mathcal{R} z ; s), n \in \mathbb{Z}$. (f) $\varphi_{m}^{n}(s)=\varphi_{-m}^{-n}(s), n, m \in \mathbb{Z}$.

(g) $\varphi_{m}^{n}(s)=\varphi_{n}^{m}(s), n, m \in \mathbb{Z}$

(h) Assume $s=1 / 2+i R$. Then for $m, n \neq 0, m \neq$ $\pm n, \operatorname{Im} \varphi_{m}^{n}(s)=\operatorname{Im} \varphi_{-m}^{n}(s)$. For $n \neq 0, \operatorname{Im} \varphi_{n}^{n}(s)-$ $\operatorname{Im} \varphi_{-n}^{n}(s)=\frac{1}{\pi} \sinh (\pi R)$.

Proof. (a) The first part is [Hejhal 83, p. 33, Proposition 6.5 (a), p. 250, Theorem $3.5(\mathrm{~b})$ ], and the second relation follows directly from $(2-5)$ for $n \neq 0$ and $\operatorname{Re} s>1$, and via meromorphic continuation to all $s \in \mathbb{C}$. For $n=0$ one uses the second part of [Hejhal 83, p. 41 (6.7)] in place of $(2-5)$.

(b) This is [Niebur 73, p. 142, Theorem 5] (see also [Hejhal 83, p. 255, Theorem $4.3(\mathrm{v})]$ ).

(c) We use $\Gamma \mathcal{R}=\mathcal{R} \Gamma$ and the fact that $k_{s}(z ; w)$ depends only on the hyperbolic distance between $z$ and $w$ $\left(\right.$ so $\left.k_{s}(\mathcal{R} z ; \mathcal{R} w)=k_{s}(z ; w)\right)$ and obtain, for $\operatorname{Re} s>1$,

$$
\begin{aligned}
G_{s}(\mathcal{R} z ; \mathcal{R} w) & =\sum_{T \in \Gamma} k_{s}(T \mathcal{R} z ; \mathcal{R} w)=\sum_{T \in \Gamma} k_{s}(\mathcal{R} T z ; \mathcal{R} w) \\
& =\sum_{T \in \Gamma} k_{s}(T z ; w)=G_{s}(z ; w)
\end{aligned}
$$

Finally, apply meromorphic continuation.

(d) This follows from (c) and the automorphy of $G_{s}(z ; w)$.

(e) This follows from (c) and (2-4).

(f) This follows from (e) and (2-6).

(g) Applied to (2-6), (a) implies $\varphi_{-m}^{-n}(s)=\overline{\varphi_{m}^{n}(\bar{s})}$. The desired result now follows from (f) and [Hejhal 83, p. 258, Theorem 5.3 (iv), Proposition 5.5 (ii)].

(h) From above, $\varphi_{m}^{n}(s)=\overline{\varphi_{m}^{n}(\bar{s})}$, which together with [Hejhal 83, p. 258, Theorem 5.3 (iii)] gives, for $s=1 / 2+$ $i R$,

$$
\begin{aligned}
& \varphi_{m}^{n}(s)-\overline{\varphi_{m}^{n}(s)} \\
& \quad=\frac{2}{\pi} \delta_{m n} \sin (\pi i R)+\frac{1}{2 s-1} \overline{\varphi_{n}^{0}(1-s)} \varphi_{m}^{0}(1-s) .
\end{aligned}
$$

When $m \neq \pm n$, the right-hand side remains unchanged if $m$ is replaced by $-m$. Thus the same holds for the lefthand side, which is just $2 i \operatorname{Im} \varphi_{m}^{n}(s)$, and the first part of the claim follows.

Now we use $m=n$ and $m=-n$ in $(2-7)$ and subtract, which yields the second part of the claim.

We now present an analogue of a Rankin-Selberg bound for $F_{n}(z ; s)$; cf., for example, [Iwaniec 02, Theorem 3.2], [Iwaniec 97, Theorem 5.1]. It will be useful for 
computational purposes to know that the Fourier coefficients of $F_{n}(z ; s)$ do not grow too rapidly. Define

$$
c_{\Gamma}=\min \left\{|c|:\left(\begin{array}{ll}
a & b \\
c & d
\end{array}\right) \in \Gamma \backslash \Gamma_{\infty}\right\} .
$$

By Shimizu's lemma [Shimizu 63, p. 42], we know that $c_{\Gamma} \geq 1$.

Theorem 2.2. Let $\Gamma$ be a cofinite Fuchsian group with one normalized cusp at $i \infty$. Let $s \in \mathbb{C} \backslash\left\{\frac{1}{2}\right\}$, s not a pole of $F_{n}(z ; s)$, and suppose $n \neq 0$. Then we have

$$
\sum_{1 \leq|m| \leq N}\left|\varphi_{m}^{n}(s)\right|^{2}=O\left(N e^{8 \pi c_{\Gamma}^{-1} \sqrt{|n| N}}\right)
$$

as $N \rightarrow \infty$. The implied constant depends on $\Gamma$, n, and $s$.

Proof. The method of proof is as in [Marklof and Strömbergsson 03, Lemma 2.7]. Let $B_{0}>1$ and let $\mathcal{F}$ be as in the third paragraph of this section and keep $0<Y<H$. We study the following integral over $\mathcal{D}=(-1 / 2,1 / 2) \times$ $(Y, H)$ :

$$
J=\int_{\mathcal{D}}\left|F_{n}(z ; s)\right|^{2} d \mu(z) .
$$

As in [Marklof and Strömbergsson 03], we tessellate $\mathcal{D}$ as $\mathcal{D}=\cup_{T \in \Gamma}(\mathcal{D} \cap T(\mathcal{F}))$ and find that $\mathcal{D}$ is covered by translates of $\mathcal{F}_{B}=\mathcal{F} \backslash[-1 / 2,1 / 2] \times[B, \infty)$ with $B=$ $\max \left(B_{0}, H,\left(c_{\Gamma}^{2} Y\right)^{-1}\right)$. (Any image $T z$ with $T \in \Gamma \backslash \Gamma_{\infty}$, $z \in \mathcal{D}$ will necessarily have $\operatorname{Im} T z<\left(c_{\Gamma}^{2} Y\right)^{-1}$; cf. $(2-8)$.) We obtain

$$
J=O\left(1+Y^{-1}\right) \int_{\mathcal{F}_{B}}\left|F_{n}(z ; s)\right|^{2} d \mu(z) .
$$

We decompose $\mathcal{F}_{B}$ as the union of $\mathcal{F}_{B_{0}}$ and $[-1 / 2,1 / 2] \times\left[B_{0}, B\right]$ and note that the above integral taken over $\mathcal{F}_{B_{0}}$ is just a constant. The Bessel asymptotics $(2-1)$ and $(2-2)$ together with (2-6) (cf. [Hejhal 83, pp. 56, 255]) leads to, for $y>B_{0}$ and $s$ fixed,

$$
F_{n}(z ; s)=O\left(e^{2 \pi|n| y}\right)
$$

To find the analogue of [Marklof and Strömbergsson 03, 2.27] we estimate

$$
\int_{B_{0}}^{B} e^{4 \pi|n| y} \frac{d y}{y^{2}} \leq\left(B-B_{0}\right) e^{4 \pi|n| B} \frac{1}{B^{2}} \leq \frac{e^{4 \pi|n| B}}{B}
$$

(since the integrand is an increasing function for $y>1$ ). Thus

$$
J=O\left(1+Y^{-1}\right) \frac{e^{4 \pi|n| B}}{B} .
$$

On the other hand, we may use Parseval's formula exactly as in the proof of [Strömbergsson 04, Proposition $4.1]$ with $F_{n}(z ; s)$ in place of $E(z ; s)$ to get

$$
J \geq \sum_{m \notin\{0, n\}}\left|\varphi_{m}^{n}(s)\right|^{2} \int_{2 \pi|m| Y}^{2 \pi|m| H}\left|K_{s-\frac{1}{2}}(y)\right|^{2} \frac{d y}{y} .
$$

We now use $K$-Bessel asymptotics to estimate the integral above. It follows from (2-1) that for large $y$ and $s$ fixed, there is a constant $c>0$ such that $\left|K_{s-1 / 2}(y)\right|>$ $c y^{-1 / 2} e^{-y}$. Thus for $|m| Y$ large enough,

$$
\begin{aligned}
\int_{2 \pi|m| Y}^{2 \pi|m| H}\left|K_{s-\frac{1}{2}}(y)\right|^{2} \frac{d y}{y} & >c \cdot \int_{2 \pi|m| Y}^{2 \pi|m| H} e^{-2 y} \frac{d y}{y^{2}} \\
& \geq c \frac{2 \pi|m|(H-Y) e^{-4 \pi|m| H}}{(2 \pi|m| H)^{2}}
\end{aligned}
$$

Choosing $Y=c_{\Gamma}^{-1} \sqrt{|n| / N}$ and $H=c_{\Gamma}^{-1} \sqrt{|n| / N}+1 / N$, we get

$$
\int_{2 \pi|m| Y}^{2 \pi|m| H}\left|K_{s-\frac{1}{2}}(y)\right|^{2} \frac{d y}{y}>c \frac{e^{-4 \pi|m|\left(c_{\Gamma}^{-1} \sqrt{\frac{|n|}{N}}+\frac{1}{N}\right)}}{2 \pi N|m|\left(c_{\Gamma}^{-1} \sqrt{\frac{|n|}{N}}+\frac{1}{N}\right)^{2}} .
$$

Consequently, there is a constant $c^{\prime}>0$ (independent of $N)$ such that for $N / 2 \leq|m| \leq N$ and $N$ large enough we have

$$
\int_{2 \pi|m| Y}^{2 \pi|m| H}\left|K_{s-\frac{1}{2}}(y)\right|^{2} \frac{d y}{y}>c^{\prime} N^{-1} e^{-4 \pi c_{\Gamma}^{-1} \sqrt{|n| N}} .
$$

Hence

$$
\sum_{N / 2 \leq|m| \leq N}\left|\varphi_{m}^{n}(s)\right|^{2} \leq J \cdot O\left(N e^{4 \pi c_{\Gamma}^{-1} \sqrt{|n| N}}\right) .
$$

Combining this with $(2-10)$ and noticing that $B=$ $\left(c_{\Gamma}^{2} Y\right)^{-1}=c_{\Gamma}^{-1} \sqrt{N /|n|}$ for $N$ large enough, we get

$$
\sum_{N / 2 \leq|m| \leq N}\left|\varphi_{m}^{n}(s)\right|^{2}=O\left(N e^{8 \pi c_{\Gamma}^{-1} \sqrt{|n| N}}\right) .
$$

The theorem now follows by standard dyadic decomposition (using the trivial bound $\left|\varphi_{m}^{n}(s)\right|^{2}=O(1)$ for each $m$ that is not large enough).

As a consequence (for each fixed $n \neq 0$ ), we have

$$
\varphi_{m}^{n}(s)=O\left(\sqrt{|m|} e^{4 \pi c_{\Gamma}^{-1} \sqrt{|n m|}}\right) \quad \text { as }|m| \rightarrow \infty .
$$

Compare [Hejhal 83, pp. 61-62] in the case $\operatorname{Re}(s)>1$.

The next theorem shows that the exponent in Theorem 2.2 is the best possible. 
Theorem 2.3. Let $\Gamma$ be a cofinite Fuchsian group with one normalized cusp at $i \infty$. Fix any $n \neq 0$ and $s \in$ $\mathbb{C} \backslash\left\{\frac{1}{2}\right\}$ not a pole of $F_{n}(z ; s)$. Then for any constants $0<\delta<4 \pi c_{\Gamma}^{-1} \sqrt{|n|}$ and $C>0$ there exist infinitely many numbers $m \in \mathbb{Z}$ such that

$$
\left|\varphi_{m}^{n}(s)\right|>C e^{\delta \sqrt{|m|}}
$$

Proof. Suppose that the claim is false, i.e., suppose that for some fixed $n \neq 0$ and some fixed $s$ as in the statement of the theorem, there exist some fixed constants $0<\delta<$ $4 \pi c_{\Gamma}^{-1} \sqrt{|n|}$ and $C>0$ such that

$$
\left|\varphi_{m}^{n}(s)\right| \leq C e^{\delta \sqrt{|m|}}
$$

holds for all but finitely many numbers $m \in \mathbb{Z}$. By increasing $C$ we will assume that $(2-12)$ holds for all $m \in \mathbb{Z}$, and we will show that this leads to a contradiction. We will assume $n>0$ (this means simply that we omit absolute value signs for $n$ throughout this proof).

Keep $y \in(0,1 / 10)$. Recall $(2-8)$, take $\left(\begin{array}{ll}a & b \\ c & d\end{array}\right) \in \Gamma$ with $c=c_{\Gamma}$, and note that $\left(\begin{array}{ll}a & b \\ c & d\end{array}\right)(i \infty)=a / c$. Using automorphy and the same reasoning that led to $(2-9)$, we get for some constant $k \neq 0($ as $y \rightarrow 0)$,

$$
\begin{aligned}
F_{n}\left(\frac{a}{c}+i y ; s\right) & =F_{n}\left(\left(\begin{array}{ll}
a & b \\
c & d
\end{array}\right)^{-1}\left(\frac{a}{c}+i y\right) ; s\right)(2-13) \\
& =F_{n}\left(-\frac{d}{c_{\Gamma}}+\frac{i}{c_{\Gamma}^{2} y} ; s\right) \sim k e^{2 \pi n /\left(c_{\Gamma}^{2} y\right)} .
\end{aligned}
$$

On the other hand we have from $(2-6)$ and $(5-1)$ in Section 5.1 that

$$
\begin{aligned}
F_{n}\left(\frac{a}{c}+i y ; s\right)= & O\left(y^{\operatorname{Re} s}\right)+O\left(y^{1-\operatorname{Re} s}\right) \\
& +O\left(\sum_{m \neq 0}\left|\varphi_{m}^{n}(s) y^{1 / 2} K_{s-\frac{1}{2}}(2 \pi|m| y)\right|\right) .
\end{aligned}
$$

Hence, using (2-12) and the bound $\left|K_{s-1 / 2}(Y)\right|<B$. $\max \left(Y^{-A}, Y^{-1 / 2}\right) e^{-Y}$ for some constants $B>0$ and $A \geq$ $1 / 2$, uniformly over all $Y>0$ (cf. $(2-1),(5-1),(5-5)$ ), we obtain

$$
\begin{aligned}
F_{n}\left(\frac{a}{c}+i y ; s\right)= & O\left(y^{\operatorname{Re} s}\right)+O\left(y^{1-\operatorname{Re} s}\right) \\
& +O\left(y^{1 / 2-A} \sum_{m \neq 0} e^{\delta \sqrt{|m|}-2 \pi|m| y}\right) .
\end{aligned}
$$

We now study the exponent in the sum above as a function of $m$; i.e., let $f(m)=\delta \sqrt{|m|}-2 \pi|m| y$. By differentiating we find that $f(m) \leq \delta^{2} /(8 \pi y)$ for all $m \in \mathbb{Z}$.
And furthermore, $f(m)<-y|m|$ when $|m|>(\delta / y)^{2}$. We obtain

$$
\begin{aligned}
F_{n}\left(\frac{a}{c}+i y ; s\right)= & O\left(y^{\operatorname{Re} s}\right)+O\left(y^{1-\operatorname{Re} s}\right) \\
& +O\left(y^{1 / 2-A}\left(\frac{\delta}{y}\right)^{2} e^{\delta^{2} /(8 \pi y)}\right. \\
& \left.\quad+y^{1 / 2-A} \sum_{m>(\delta / y)^{2}} e^{-y m}\right) \\
= & O\left(y^{-3 / 2-A} e^{\delta^{2} /(8 \pi y)}\right) \text { as } y \rightarrow 0 .
\end{aligned}
$$

Since our assumption $0<\delta<4 \pi c_{\Gamma}^{-1} \sqrt{n}$ implies that $\delta^{2} /(8 \pi)<2 \pi c_{\Gamma}^{-2} n$, this contradicts $(2-13)$.

\section{COMPUTATIONS OF $\boldsymbol{F}_{n}(z ; s)$}

We will base our computations of $F_{n}(z ; s)$ and $G_{s}(z ; w)$ on the algorithm developed by Hejhal for computations of cusp forms; cf. [Hejhal 99]. It was adapted for the Eisenstein series in [Avelin 07], and considering (2-6) (and the fact that $F_{0}(z ; s)=E(z ; s)$ ), it should also be possible to adjust it to $F_{n}(z ; s)$ without much effort. Regarding the algorithm itself, this is true. However, while previous computations of cusp forms and Eisenstein series have already prodded the development of a stable routine for the $K$-Bessel function, cf. [Hejhal 92, Avelin 07], the $I$-Bessel function introduces a new computational difficulty for $F_{n}(z ; s)$. Computations of Bessel functions will be discussed further in Section 5 .

To present the main ideas of the algorithm (which will be useful to have in mind when $G_{s}(z ; w)$ is treated in Section 4) we will repeat parts of [Avelin 07, Section 4.1] for the more general functions $F_{n}(z ; s), n \in \mathbb{Z}$ (with $\chi=1)$.

In principle, a bound like Theorem 2.2 enables us to truncate the Fourier series at some $m=M(y)$ (a monotonically decreasing function) with an error $R_{M(y)}$ whose absolute value is less than some prescribed small number:

$$
F_{n}(z ; s)=\alpha_{n}(z)+\sum_{m=-M(y)}^{M(y)} c_{m}(y) e^{2 \pi i m x}+R_{M(y)} .
$$

Here we use

$\alpha_{n}(z)= \begin{cases}A_{n}(y) e^{2 \pi i n x}=y^{1 / 2} I_{s-\frac{1}{2}}(2 \pi|n| y) e^{2 \pi i n x}, & n \neq 0, \\ A_{0}(y)=y^{s}, & n=0,\end{cases}$

$c_{m}(y)=\varphi_{m}^{n}(s) k_{m}(y), \quad k_{0}(y)=\frac{y^{1-s}}{2 s-1}$,

$k_{m}(y)=y^{1 / 2} K_{s-\frac{1}{2}}(2 \pi|m| y), \quad$ for $m \neq 0$. 
Let $Y_{\min }=\min \{\operatorname{Im}(z): z \in \mathcal{F}\}$ (cf. Section 2), set $M_{0}=M\left(Y_{\min }\right)$, and choose any $Y<Y_{\min }$. We then take $Q \geq M(Y)+1$ and $2 Q$ equally spaced points

$$
z_{j}=x_{j}+i Y, \quad \text { where } x_{j}=\frac{j-1 / 2}{2 Q}, j=1-Q, \ldots, Q .
$$

These points are used in a finite Fourier transform to solve for $\varphi_{m}^{n}(s)$ for $-M_{0} \leq m \leq M_{0}$ (from this point the error term is neglected):

$$
\frac{1}{2 Q} \sum_{j=1-Q}^{Q} F_{n}\left(z_{j} ; s\right) e^{-2 \pi i m x_{j}}=\delta_{m n} A_{n}(Y)+c_{m}(Y) .
$$

To dissolve the above tautology we use automorphy: $F_{n}\left(z_{j} ; s\right)=F_{n}\left(z_{j}^{*} ; s\right)$, where $z_{j}^{*}$ denotes the image of $z_{j}$ in $\mathcal{F}$. This leads to

$$
\begin{gathered}
\delta_{m n} A_{n}(Y)+c_{m}(Y)=\frac{1}{2 Q} \sum_{j=1-Q}^{Q} F_{n}\left(z_{j}^{*} ; s\right) e^{-2 \pi i m x_{j}} \\
=\frac{1}{2 Q} \sum_{j=1-Q}^{Q}\left(A_{n}\left(y_{j}^{*}\right) e^{2 \pi i n x_{j}^{*}}\right. \\
\left.\quad+\sum_{k=-M_{0}}^{M_{0}} c_{k}\left(y_{j}^{*}\right) e^{2 \pi i k x_{j}^{*}}\right) e^{-2 \pi i m x_{j}} \\
=\frac{1}{2 Q} \sum_{j=1-Q}^{Q} A_{n}\left(y_{j}^{*}\right) e^{2 \pi i n x_{j}^{*}} e^{-2 \pi i m x_{j}} \\
+\frac{1}{2 Q} \sum_{\ell=-M_{0}}^{M_{0}} \varphi_{\ell}^{n}(s) \sum_{j=1-Q}^{Q} k_{\ell}\left(y_{j}^{*}\right) e^{2 \pi i \ell x_{j}^{*}} e^{-2 \pi i m x_{j}} .
\end{gathered}
$$

The final system of equations is, for $m=-M_{0}, \ldots, M_{0}$,

$$
\sum_{\ell=-M_{0}}^{M_{0}} \varphi_{\ell}^{n}(s) \widetilde{V}_{m \ell}=-\widetilde{A}_{m n}
$$

where

$$
\begin{aligned}
& \widetilde{A}_{m n}=\frac{1}{2 Q} \sum_{j=1-Q}^{Q} A_{n}\left(y_{j}^{*}\right) e^{2 \pi i n x_{j}^{*}} e^{-2 \pi i m x_{j}}-\delta_{m n} A_{n}(Y), \\
& \widetilde{V}_{m \ell}=\frac{1}{2 Q} \sum_{j=1-Q}^{Q} k_{\ell}\left(y_{j}^{*}\right) e^{2 \pi i \ell x_{j}^{*}} e^{-2 \pi i m x_{j}}-\delta_{m \ell} k_{m}(Y) .
\end{aligned}
$$

Each permitted choice of $Y$ and $M_{0}$ should yield the same result.

Just as in [Hejhal 99, Avelin 07], it is convenient to use a rescaled version of $K_{s-1 / 2}(x)$, and likewise with $I_{s-1 / 2}(x)$. More precisely, motivated by $(2-4)$ above, we actually work with

$$
\begin{aligned}
e^{-R \pi / 2} & F_{n}(z ; s) \\
= & y^{1 / 2} \widehat{I}_{s-\frac{1}{2}}(2 \pi|n| y) e^{2 \pi i n x}+e^{-R \pi / 2} \varphi_{0}^{n}(s) \frac{y^{1-s}}{2 s-1} \\
& +\sum_{m \neq 0} e^{-R \pi} \varphi_{m}^{n}(s) y^{1 / 2} \widehat{K}_{s-\frac{1}{2}}(2 \pi|m| y) e^{2 \pi i m x}
\end{aligned}
$$

where $R=\operatorname{Im} s$ and

$$
\widehat{I}_{s-\frac{1}{2}}(x)=e^{-R \pi / 2} I_{s-\frac{1}{2}}(x), \quad \widehat{K}_{s-\frac{1}{2}}(x)=e^{R \pi / 2} K_{s-\frac{1}{2}}(x) .
$$

We assume herein that $\operatorname{Im} s \geq 0$.

\section{COMPUTATIONS OF $G_{s}(z ; w)$}

Because $G_{s}(z ; w)$ is automorphic and $\Gamma$ is normalized to have $\Gamma_{\infty}=[S]$, we can choose $w=u+i v$ such that

$$
v=\max \{\operatorname{Im} T w: T \in \Gamma\}
$$

and $-1 / 2<u \leq 1 / 2$. We will assume that this has been done and regard $w$ as fixed. Write $G_{s}(z ; w)$ with $z=x+i y$ as

$$
\begin{aligned}
& G_{s}(z ; w) \\
& \quad=\frac{E(w ; s) y^{1-s}}{1-2 s}+\sum_{m \neq 0} f_{m} y^{1 / 2} \widehat{K}_{s-\frac{1}{2}}(2 \pi|m| y) e^{2 \pi i m x}
\end{aligned}
$$

with

$$
f_{m}=-e^{-R \pi / 2} F_{-m}(w ; s) .
$$

This Fourier development is convergent whenever $y>v$, and it diverges otherwise (cf. [Hejhal 83, Section 6.6]). This is certainly a problem if one would like to use the algorithm described in Section 3. Actually, this algorithm relies on the fact that the same Fourier expansion is valid below the fundamental region as well as inside the fundamental region. Therefore, some additional work is necessary before the algorithm is applicable.

Let $\Gamma_{w}$ be the stabilizer of $w$. Near $z=w$ we have

$$
G_{s}(z ; w)=\frac{\mu}{2 \pi} \ln |z-w|+O(1)
$$

where $\mu=\# \Gamma_{w}$; cf. [Hejhal 83, p. 34]. The idea is to subtract off from $G_{s}(z ; w)$ the bad behavior at $z=w$ to get a function that has a Fourier expansion valid in a larger region. In view of $(2-3)$ one would like to take, for $\operatorname{Re} s>1$,

$$
\widetilde{G}_{s}(z ; w)=G_{s}(z ; w)-\mu \sum_{n \in \mathbb{Z}} k_{s}(z ; w+n) .
$$


What we have done here is essentially to have subtracted off the entire row of singularities $w+n, n \in \mathbb{Z}$. Then $\widetilde{G}_{s}(z ; w)$ is not automorphic, but it is an eigenfunction of $\Delta$ with the same eigenvalue as $G_{s}(z ; w)$ and it is bounded near $z=w+n$. It will have a Fourier expansion valid for $y>v_{1}$, where $v_{1}=\sup \left\{\operatorname{Im}(T w): T \in \Gamma \backslash \Gamma_{\infty}\right\}$. This will be sufficient as long as we can find a suitable $Y>v_{1}$. Otherwise, additional levels of singularities must be subtracted off.

We will now explain this procedure in detail. Let $D_{w}=\{w+n: n \in \mathbb{Z}\}$ and define

$$
P_{s}(z ; w)=\sum_{n \in \mathbb{Z}} k_{s}(z ; w+n)
$$

for $z \notin D_{w}$. From [Hejhal 83, p. 31, Proposition 6.2] we know this sum to be nicely convergent for $\operatorname{Re} s>1$. But it is actually clear from the proof of [Hejhal 83, Proposition 6.2 that it is convergent for $\operatorname{Re} s>1 / 2$ if we use instead of [Hejhal 83, Proposition 5.1] the fact that our sum is only over $T \in \Gamma_{\infty}$.

Fourier coefficients for $\sum_{n \in \mathbb{Z}} k_{s}(z ; w+n)$ are found in [Hejhal 83, p. 35]. We have, for $\operatorname{Re} s>1 / 2$,

$$
\begin{aligned}
& P_{s}(z ; w) \\
& =\left\{\begin{array}{cc}
\frac{v^{s} y^{1-s}}{1-2 s}-\sum_{m \neq 0} e^{-2 \pi i m u} v^{1 / 2} \widehat{I}_{s-\frac{1}{2}}(2 \pi|m| v) \\
\quad \times y^{1 / 2} \widehat{K}_{s-\frac{1}{2}}(2 \pi|m| y) e^{2 \pi i m x} & \text { for } y>v, \\
\frac{y^{s} v^{1-s}}{1-2 s}-\sum_{m \neq 0} e^{-2 \pi i m u} y^{1 / 2} \widehat{I}_{s-\frac{1}{2}}(2 \pi|m| y) \\
\times v^{1 / 2} \widehat{K}_{s-\frac{1}{2}}(2 \pi|m| v) e^{2 \pi i m x} & \text { for } y<v .
\end{array}\right.
\end{aligned}
$$

(Note that the two versions are symmetric with respect to $y$ and $v$.)

We will now make some remarks about the convergence of the sums in (4-6). Fix $\varepsilon>0$ and keep $|z-(w+n)|>\varepsilon$ for all $n \in \mathbb{Z}$, and $\operatorname{Im} z>\varepsilon$. Assuming $|m|$ large enough, $(2-1)$ and $(2-2)$ give (recall (3-5))

$$
\begin{aligned}
& v^{1 / 2} y^{1 / 2} \widehat{I}_{s-\frac{1}{2}}(2 \pi|m| v) \widehat{K}_{s-\frac{1}{2}}(2 \pi|m| y) \\
& =\frac{1}{4 \pi|m|} e^{-2 \pi|m|(y-v)}\left(1+O\left(\frac{1}{|m|}\right)\right),
\end{aligned}
$$

and so the first sum in $(4-6)$ is

$$
C \cdot \sum_{m \neq 0} \frac{1}{|m|} e^{-2 \pi|m|(y-v)}\left(1+O\left(\frac{1}{|m|}\right)\right) e^{2 \pi i m(x-u)},
$$

for some constant $C$. This is absolutely convergent when $y>v$, conditionally convergent when $y=v$, and the convergence is uniform over our set of $z$-values. Moreover, when $y$ is near $v$, the sum above is essentially bounded (up to a constant) by

$$
\begin{aligned}
1+ & \int_{1}^{\infty} x^{-1} e^{-2 \pi x(y-v)} d x \\
& =1+\int_{2 \pi(y-v)}^{\infty} t^{-1} e^{-t} d t=\int_{2 \pi(y-v)}^{A} t^{-1} e^{-t} d t+C_{1} \\
& <\int_{2 \pi(y-v)}^{A} t^{-1} d t+C_{1}=-\ln (2 \pi(y-v))+C_{2},
\end{aligned}
$$

where $C_{1}, C_{2}, A$ are some positive numbers. By symmetry, we conclude that both series (4-6) are uniformly convergent when $|z-(w+n)|>\varepsilon$ for all $n \in \mathbb{Z}$ and $y>\varepsilon$, and that they have a logarithmic bound when $y$ is near $v$.

After these considerations it seems likely that the key to realizing (4-4) for general $s \in \mathbb{C}$ is the Fourier expansions $(4-6)$. In the following lemma we redefine $P_{s}(z ; w)$ and show that it has the right properties.

Lemma 4.1. Let $w \in \mathcal{H}$ be fixed, $z \in \mathcal{H}$, and $s \in \mathbb{C} \backslash$ $\left\{\frac{1}{2}, 0,-1,-2,-3, \ldots\right\}$. Take $(4-6)$ as the definition of $P_{s}(z ; w)$. Then $P_{s}(z ; w)$, regarded as a function of $z$, is $C^{\infty}$ whenever $z \notin D_{w}$; it satisfies $\Delta_{z} f+s(1-s) f=0$; and

near $z=w$.

$$
P_{s}(z ; w)=\frac{1}{2 \pi} \ln |z-w|+O(1)
$$

Proof. A hyperbolic open disk with radius $\varepsilon$ and center $z$ will be denoted by $N_{\varepsilon}(z)$.

First fix $z_{0} \notin D_{w}$ and choose $\varepsilon>0$ such that $N_{2 \varepsilon}\left(z_{0}\right) \cap$ $D_{w}=\emptyset$. We will show that $P_{s}(z ; w)$ is $C^{\infty}$ and satisfies $\Delta_{z} f+s(1-s) f=0$ for $z \in N_{\varepsilon}\left(z_{0}\right)$.

Let $\Phi\left(\frac{|\zeta-z|^{2}}{\operatorname{Im} \zeta \operatorname{Im} z}\right)$ be a smooth bump function that is nonzero only when $\zeta \in N_{\varepsilon}(z)$ (note that $\Phi$ is a function of the hyperbolic distance between $z$ and $\zeta$ ).

For a moment take $\operatorname{Re} s>1 / 2\left(\right.$ then $P_{s}(z ; w)$ satisfies $(4-5)$ and $\left.\Delta_{z} f+s(1-s) f=0\right)$ and use [Hejhal 76, p. 8, Proposition 3.1] to find that

$$
\int_{\mathcal{H}} \Phi\left(\frac{|\zeta-z|^{2}}{\operatorname{Im} \zeta \operatorname{Im} z}\right) P_{s}(\zeta ; w) d \mu(\zeta)=\Lambda(s(1-s)) P_{s}(z ; w)
$$

holds for all $z \in N_{\varepsilon}\left(z_{0}\right)$ with $\Lambda$ depending only on $s$ and $\Phi$. For any given compact set $K \subset \mathbb{C}$ we may choose $\Phi$ in such a way that $\Lambda(s(1-s))$ is nonzero for all $s \in K$; cf., for example, [Iwaniec 02, pp. 99ff.]. From (4-6), each side of (4-8) is analytic in $s$ for all $s \in \mathbb{C} \backslash\left\{\frac{1}{2}\right\}$, and so by analytic continuation, the equality holds for all $s \in$ $\mathbb{C} \backslash\left\{\frac{1}{2}\right\}$.

The left-hand side of (4-8) is clearly $C^{\infty}$ for all $z \in$ $N_{\varepsilon}\left(z_{0}\right)$. We have $\Delta_{z} \Phi\left(\frac{|\zeta-z|^{2}}{\operatorname{Im} \zeta \operatorname{Im} z}\right)=\Phi_{1}\left(\frac{|\zeta-z|^{2}}{\operatorname{Im} \zeta \operatorname{Im} z}\right)$, where 
$\Phi_{1}$ is another smooth function that is nonzero only when $\zeta \in N_{\varepsilon}(z)$, for which a relation analogous to (4-8) is true with a function $\Lambda_{1}$ in place of $\Lambda$; cf. [Selberg 56, p. 52]. Moreover, it follows from

$$
\Delta_{\zeta} \Phi\left(\frac{|\zeta-z|^{2}}{\operatorname{Im} \zeta \operatorname{Im} z}\right)=\Delta_{z} \Phi\left(\frac{|\zeta-z|^{2}}{\operatorname{Im} \zeta \operatorname{Im} z}\right)
$$

cf. [Selberg 56, p. 52], that

$$
\Lambda_{1}(s(1-s))=-s(1-s) \Lambda(s(1-s)) .
$$

Now the desired properties of $P_{s}(z ; w)$ for $z \in N_{\varepsilon}\left(z_{0}\right)$ follow: we obtain that $P_{s}(z ; w)$ is $C^{\infty}$ in $z$ for all $z \in$ $N_{\varepsilon}\left(z_{0}\right)$, and

$$
\Delta_{z} P_{s}(z ; w)=-s(1-s) P_{s}(z ; w)
$$

It remains to prove the logarithmic bound. Take $\varepsilon$ such that $N_{2 \varepsilon}(w) \cap D_{w}=\{w\}$. We will show that $P_{s}(z ; w)-k_{s}(z ; w)$ is bounded when $z$ is near $w$. Take $z \in N_{\varepsilon}(w) \backslash\{w\}$. Again let $\Phi\left(\frac{|\zeta-z|^{2}}{\operatorname{Im} \zeta \operatorname{Im} z}\right)$ be a smooth bump function that is nonzero only in $N_{\varepsilon}(z)$, and find exactly as above that for $\operatorname{Re} s>1 / 2$,

$$
\begin{gathered}
\int_{\mathcal{H}} \Phi\left(\frac{|\zeta-z|^{2}}{\operatorname{Im} \zeta \operatorname{Im} z}\right)\left(P_{s}(\zeta ; w)-k_{s}(\zeta ; w)\right) d \mu(\zeta) \\
=\Lambda(s(1-s))\left(P_{s}(z ; w)-k_{s}(z ; w)\right)
\end{gathered}
$$

holds for all $z \in N_{\varepsilon}(w) \backslash\{w\}$.

Recall from $(4-7)$ that $\left|P_{s}(\zeta ; w)\right| \leq O(-\ln |\operatorname{Im} \zeta-v|)$ for $\operatorname{Im} \zeta$ near $v$, and one checks that this holds uniformly for $s$ in compact subsets of $\mathbb{C} \backslash\{1 / 2\}$. The same bound holds for $k_{s}(\zeta ; w)$, for $s \in \mathbb{C} \backslash\{0,-1,-2,-3, \ldots\}$; cf. Section 2.

Therefore the integral on the left-hand side of (4-9) is analytic in $s$ for $s \in \mathbb{C} \backslash\left\{\frac{1}{2}, 0,-1,-2,-3, \ldots\right\}$. The righthand side is analytic for the same set of $s$-values. Thus we may once more use analytic continuation and conclude that (4-9) holds for $s \in \mathbb{C} \backslash\left\{\frac{1}{2}, 0,-1,-2,-3, \ldots\right\}$. The left-hand side of (4-9) is bounded if $z$ approaches $w$, and it follows that so is the right-hand side.

We will now show a more general version of (4-4). Let $v_{0}=\operatorname{Im} w$, and define, for $k=0,1,2, \ldots$,

$$
\begin{aligned}
v_{k+1} & =\max \left\{\operatorname{Im} T w: T \in \Gamma, \operatorname{Im} T w<v_{k}\right\}, \\
H_{v_{k}} & =\left\{T w: T \in \Gamma, \operatorname{Im} T w=v_{k},-\frac{1}{2}<\operatorname{Re} T w \leq \frac{1}{2}\right\} .
\end{aligned}
$$

Note that $v_{0}=v$ and recall $(4-1)$. Define, for $s \in \mathbb{C} \backslash$ $\left\{\frac{1}{2}, 0,-1,-2,-3, \ldots\right\}, r \in \mathbb{Z}^{+}$,

$$
G_{s}^{r}(z ; w)=G_{s}(z ; w)-\mu \sum_{k=0}^{r} \sum_{w^{\prime} \in H_{v_{k}}} P_{s}\left(z ; w^{\prime}\right) .
$$

Note that by $(4-6)$ we have for $y>v_{0}$ (with $w^{\prime}=u^{\prime}+$ $\left.i v_{k}\right)$,

$$
\begin{aligned}
& G_{s}^{r}(z ; w)=\frac{\left(E(w ; s)-\mu \sum_{k=0}^{r} \sum_{w^{\prime} \in H_{v_{k}}} v_{k}^{s}\right) y^{1-s}}{1-2 s} \\
& +\sum_{m \neq 0}\left(f_{m}+\mu \sum_{k=0}^{r} \sum_{w^{\prime} \in H_{v_{k}}} e^{-2 \pi i m u^{\prime}} v_{k}^{1 / 2} \widehat{I}_{s-\frac{1}{2}}\left(2 \pi|m| v_{k}\right)\right) \\
& \quad \times y^{1 / 2} \widehat{K}_{s-\frac{1}{2}}(2 \pi|m| y) e^{2 \pi i m x}
\end{aligned}
$$

However, by its definition, (4-10), and using Lemma 4.1, $G_{s}^{r}(z ; w)$ is in fact a $C^{\infty}$ function of $z$, and it satisfies $\Delta_{z} f+s(1-s) f=0$ in the entire region $y>v_{r+1}$. Hence by [Hejhal 83, p. 23, Proposition 4.10], the above Fourier expansion $(4-11)$ is valid for all $y>v_{r+1}$.

We introduce some simplifying notation and write (4-11) as

$$
G_{s}^{r}(z ; w)=\Psi^{r}(y)+\sum_{m \neq 0} f_{m}^{r} y^{1 / 2} \widehat{K}_{s-\frac{1}{2}}(2 \pi|m| y) e^{2 \pi i m x}
$$

Our preparations are now finished. We will proceed with the algorithm presented in Section 3 applied to the function $G_{s}^{r}(z ; w)$. We begin by truncating. Using [Hejhal 83, p. 23, Proposition 4.10] (with $A=v_{r+1}$ ), we have for a fixed, small $\varepsilon>0$,

$$
f_{m}^{r}=O\left(\frac{e^{2 \pi|m|\left(v_{r+1}+\varepsilon\right)}}{\sqrt{|m|}}\right)
$$

This bound leads us to expect to find a reasonable $M(y)$ for all $y$ sufficiently separated from $v_{r+1}$ such that

$$
G_{s}^{r}(z ; w)=\Psi^{r}(y)+\sum_{\substack{m=-M(y) \\ m \neq 0}}^{M(y)} c_{m}(y) e^{2 \pi i m x}+S_{M(y)}
$$

with $c_{m}(y)=f_{m}^{r} y^{1 / 2} \widehat{K}_{s-1 / 2}(2 \pi|m| y)$ and sufficiently small error term $S_{M(y)}$. For $1 \leq|n| \leq M_{0}$ (with $z_{j}=x_{j}+i Y$ as in (3-2)), the analogue of $(3-3)$ is

$$
\begin{aligned}
& \frac{1}{2 Q} \sum_{j=1-Q}^{Q}\left(\Psi^{r}(Y)+\sum_{\substack{m=-M(Y) \\
m \neq 0}}^{M(Y)} c_{m}(Y) e^{2 \pi m x_{j}}\right) e^{-2 \pi n x_{j}} \\
& \quad=c_{n}(Y) .
\end{aligned}
$$

The next step is to use automorphy. Recall that $G_{s}^{r}(z ; w)$ is unfortunately not automorphic. However, 
$G_{s}(z ; w)$ is automorphic in $z$, which is equivalent to

$$
\begin{aligned}
& G_{s}^{r}\left(z_{j} ; w\right)+\mu \sum_{k=0}^{r} \sum_{w^{\prime} \in H_{v_{k}}} P_{s}\left(z_{j} ; w^{\prime}\right) \\
& =G_{s}^{r}\left(z_{j}^{*} ; w\right)+\mu \sum_{k=0}^{r} \sum_{w^{\prime} \in H_{v_{k}}} P_{s}\left(z_{j}^{*} ; w^{\prime}\right) .
\end{aligned}
$$

Substituting this in (4-14), we obtain

$$
\begin{aligned}
& \frac{1}{2 Q} \sum_{j=1-Q}^{Q}\left(\Psi^{r}\left(y_{j}^{*}\right)\right. \\
& \left.\quad+\mu \sum_{k=0}^{r} \sum_{w^{\prime} \in H_{v_{k}}}\left(P_{s}\left(z_{j}^{*} ; w^{\prime}\right)-P_{s}\left(z_{j} ; w^{\prime}\right)\right)\right) e^{-2 \pi n x_{j}} \\
& \quad+\frac{1}{2 Q} \sum_{\substack{m=-M_{0} \\
m \neq 0}}^{M_{0}} \sum_{j=1-Q}^{Q} c_{m}\left(y_{j}^{*}\right) e^{2 \pi m x_{j}^{*}} e^{-2 \pi n x_{j}}=c_{n}(Y) .
\end{aligned}
$$

We choose the appropriate Fourier representation for each $P_{s}\left(\cdot ; w^{\prime}\right)$; cf. (4-6). Introducing the notation $A_{n}^{r}$ for the first line in the equation above, we get

$$
\begin{array}{r}
A_{n}^{r}+\sum_{\substack{m=-M_{0} \\
m \neq 0}}^{M_{0}} f_{m}^{r} \frac{1}{2 Q} \sum_{j=1-Q}^{Q} y_{j}^{* 1 / 2} \widehat{K}_{s-\frac{1}{2}}\left(2 \pi|m| y_{j}^{*}\right) \\
\times e^{2 \pi m x_{j}^{*}} e^{-2 \pi n x_{j}}=c_{n}(Y) .
\end{array}
$$

We may thus compute Fourier coefficients of $G_{s}(z ; w)$ through the following linear system of equations for $1 \leq$ $|n| \leq M_{0}$ :

$$
A_{n}^{r}+\sum_{\substack{m=-M_{0} \\ m \neq 0}}^{M_{0}} f_{m}^{r} \widetilde{V}_{n m}=0
$$

with

$$
\begin{aligned}
\widetilde{V}_{n m}= & \frac{1}{2 Q} \sum_{j=1-Q}^{Q} y_{j}^{* 1 / 2} \widehat{K}_{s-\frac{1}{2}}\left(2 \pi|m| y_{j}^{*}\right) e^{2 \pi m x_{j}^{*}} e^{-2 \pi n x_{j}} \\
& -\delta_{n m} Y^{1 / 2} \widehat{K}_{s-\frac{1}{2}}(2 \pi|m| Y)
\end{aligned}
$$

The value of the Eisenstein series $E(w ; s)$ (incorporated in $A_{n}^{r}$ ) may be computed on general one-cusp groups with the algorithm in [Avelin 07].

\section{COMPUTATIONS OF BESSEL FUNCTIONS OF IMAGINARY ORDER}

Our algorithms for computing $G_{s}(z ; w)$ and $F_{n}(z ; w)$ rely on the ability to compute the $K$ - and $I$-Bessel functions accurately. The implementation of our algorithms was done with the programming language and program library PARI/GP, and we used the built-in $K$-Bessel routine. $^{1}$

Computations of Bessel functions of complex order are more technical than computations of Bessel functions of purely imaginary order; see, for example, [Avelin 07, Section 4.3] and Section 5.1. For our present purposes it is sufficient to consider $s$-values with $\operatorname{Re} s=1 / 2$ and Bessel functions with purely imaginary order. We compute $I_{i R}(x)$ for small $x>0$ with a power series expansion combined with $K_{i R}(x)$-values. For large $x$, both $I_{i R}(x)$ and $K_{i R}(x)$ are computed using asymptotic expansions. Here we will explain our computations in greater detail. (See [Gil et al. 02, Gil et al. 03, Gil et al. 04a] for more discussion on methods for computing $I_{i R}(x)$ and $K_{i R}(x)$.)

\subsection{Method 1: Power Series}

The function $I_{\nu}(x)$ has a power series expansion around the point $x=0$ :

$$
I_{\nu}(x)=x^{\nu} \sum_{n=0}^{\infty} \frac{\left(x^{2} / 4\right)^{n}}{2^{\nu} n ! \Gamma(\nu+n+1)}
$$

where $\Gamma(a)$ denotes the $\Gamma$-function. To simplify computations, (5-1) is rewritten for $\nu=i R$ (note that it follows from Stirling's formula, cf. [Abramowitz and Stegun 64, p. 257], that $\frac{e^{-R \pi / 2}}{\Gamma(1+i R)} \sim(2 \pi R)^{-1 / 2}$ as $\left.R \rightarrow \infty\right)$ as

$$
\widehat{I}_{i R}(x)=\left(\frac{x}{2}\right)^{i R} \frac{e^{-R \pi / 2}}{\Gamma(1+i R)} \sum_{n=0}^{\infty} \beta(n)
$$

with

$$
\beta(0)=1, \quad \beta(n)=\frac{(x / 2)^{2}}{n(n+i R)} \beta(n-1) .
$$

Recall (3-5) for $\widehat{I}_{i R}(x)$. We recursively generate $\beta(n)$ until $|\beta(n)|<\varepsilon$ (for some appropriate $\varepsilon$; cf. Section 5.3).

Unfortunately, the power series (5-2) suffers catastrophic cancellation in the imaginary part, so it may be used only for computing $\operatorname{Re} I_{i R}(x) ; \operatorname{Im} I_{i R}(x)$ will simply not have enough accuracy (unless we use extremely high precision). The fact that $\operatorname{Im} I_{i R}(x)$ is in general much smaller than $\operatorname{Re} I_{i R}(x)$ can be seen from (5-1) and from (5-4) below.

However, we may compute $\operatorname{Im} I_{i R}(x)$ via $K_{i R}(x)$ (for $R \in \mathbb{R})$ using

$$
\operatorname{Im} I_{i R}(x)=-\frac{1}{\pi} \sinh (\pi R) K_{i R}(x),
$$

\footnotetext{
${ }^{1}$ Samples of our code may be downloaded from http://www .math.uu.se/research/archive/avelin/.
} 
which follows from the relation (using $\nu=i R$ and $\left.I_{\bar{\nu}}(x)=\overline{I_{\nu}(x)}\right)$

$$
K_{\nu}(x)=\frac{\pi\left(I_{-\nu}(x)-I_{\nu}(x)\right)}{2 \sin (\pi \nu)} ;
$$

cf. [Abramowitz and Stegun 64, p. 375]. Assuming that we are able to compute $K_{i R}(x)$ accurately, the imaginary part of $I_{i R}(x)$ is no longer a problem.

\subsection{Method 2: Asymptotic Expansion}

The expansions (2-1) and (2-2) are sometimes suitable for numerical computations when $x$ is large. In $(2-2)$ we write (and similarly with $(2-1)$ )

$$
\sum_{n=0}^{\infty} \frac{(-1)^{n} A_{n}(\nu)}{x^{n}}=\sum_{n=0}^{\infty} \alpha(n)
$$

with

$$
\alpha(0)=1, \quad \alpha(n)=-\frac{\nu^{2}-(n-1 / 2)^{2}}{2 n x} \alpha(n-1) .
$$

Since $(2-2)$ is an asymptotic expansion, we know that for each fixed $N$ the error $E(x, N)$ is

$$
\begin{aligned}
E(x, N) & =\left|I_{\nu}(x)-\frac{e^{x}}{\sqrt{2 \pi x}} \sum_{n=0}^{N} \frac{(-1)^{n} A_{n}(\nu)}{x^{n}}\right| \\
& =O\left(x^{-N-3 / 2}\right), \quad \text { as } x \rightarrow \infty,
\end{aligned}
$$

and similarly for $K_{\nu}(x)$. But for fixed $x, E(x, N)$ does not approach zero as $N \rightarrow \infty$. In fact, it diverges. The same is true for $K_{\nu}(x)$. Still, $(2-1)$ and $(2-2)$ provide useful approximations as long as $x$ is large enough and we use optimal truncation; cf. Section 5.3.

\subsection{Computational Details}

Here we will discuss in what ranges methods 1 and 2 may be used for $\nu=i R$ and where to truncate the infinite sums in these methods for $R \leq 200$. To some extent, it will depend on what precision we are working with. (With PARI/GP we are able to choose the precision. It is also possible to temporarily raise the precision for a part of the computation.) Denote by $P$ the working precision and let $\varepsilon$ denote a positive number that is small in relation to $P$. We use $\varepsilon=10^{-P}$.

First note that $(2-2)$ is a real approximation of $I_{i R}(x)$, so method 2 should not be used unless $\operatorname{Im} I_{i R}(x)$ is sufficiently small. We will not use method 2 unless $x>P \log 10+\frac{R \pi}{2}$ (then it turns out that $\left|\operatorname{Im} \widehat{I}_{i R}(x)\right|$ does not exceed $\varepsilon$ by much; cf. (5-4) and (2-1)).
We now study the recurrence relations (5-3) and (5-6), and we write them as $\beta(n)=d(n) \beta(n-1)$, $\alpha(n)=c(n) \alpha(n-1)$. The function

$$
c(n)=\frac{R^{2}+(n-1 / 2)^{2}}{2 n x},
$$

for $n \geq 1$, is positive, and $c(n)<1$ holds if and only if

$$
\frac{1}{2}+x-\sqrt{x^{2}-R^{2}+x}<n<\frac{1}{2}+x+\sqrt{x^{2}-R^{2}+x} .
$$

Hence the function $|\alpha(n)|$ (for $n \in \mathbb{Z}^{+}$) reaches a minimum as $n$ passes the point $\frac{1}{2}+x+\sqrt{x^{2}-R^{2}+x}$; for larger $n$ the function $|\alpha(n)|$ is increasing, and tends to $\infty$ as $n \rightarrow \infty$.

The typical behavior of a divergent asymptotic series is that the error decreases with the number of terms, $N$, until a certain optimal $N_{\text {opt }}$ is reached, and then the error increases as more terms are added. This optimal truncation is usually just before the absolute value of the terms reaches a minimum, and this seems to be true also with our series; see, for example, [Boyd 05, p. 557] and [Bender and Orszag 99, pp. 94, 122].

Therefore we truncate at the nearest integer $N$ less than $\frac{1}{2}+x+\sqrt{x^{2}-R^{2}+x}$, or when $|\alpha(n)|<\varepsilon$ if that happens for $n<N$. The crucial point here is that there is an upper limit to the accuracy of our approximations of $K_{i R}(x)$ and $I_{i R}(x)$ depending on $x$ and $R$.

We need to make certain that the error caused by this upper limit does not exceed $\varepsilon$ by too much. Test runs seem to suggest that this can be ensured by imposing the extra condition $x>6 R+24$, at least for moderate $P$. Hence, we take as our switching condition

$$
\begin{aligned}
& X=\max \left\{6 R+24, P \log 10+\frac{R \pi}{2}\right\}, \\
& \text { method } 1 \text { if } x \leq X, \\
& \text { method } 2 \text { if } x>X .
\end{aligned}
$$

The routines have been tested using spot checks against Maple computations (which are slow but known to be accurate) to rule out any intrinsic errors, and then, more extensively, we performed internal PARI/GP tests against higher precision. These suggest that $I_{i R}(x)$ for $x>X, R \leq 200$, is computed with at least $P-5$ correct significant digits; $K_{i R}(x)$ is computed with at least $P-6$ correct significant digits for $x>X, R \leq 100$, and with at least $P-13$ correct significant digits for $x>X, R \leq 200$. (These numbers are based on tests with 150,000 $x$-values for each one of five different $R$-values, and this was repeated in precisions $P=19,38,57$.) Better accuracy for 
$I_{i R}(x)$ than for $K_{i R}(x)$ was to be expected; cf. [Gil et al. 02, Gil et al. 04b].

We now turn to method 1 , the power series approximation. We find that $|d(n)|=x^{2} /(4 n|n+i R|)$ is decreasing as $n$ increases and $|d(1)| \approx x^{2} /(4 R)$. If $|d(1)|$ is greater than 1 , then $|\beta(n)|$ will reach a maximum value (when $|d(n)|$ is near 1 ) before decreasing to 0 . If the maximum value of $|\beta(n)|$ is large compared to the final result, then some significant digits will be lost. A quick fix is to raise the precision compared to the working precision in computing the power series approximation. The larger the value of $R$, the higher the precision that is needed. This is because $\max _{x} \leq X \frac{x^{2}}{4 R}$ grows with $R$, and because a large $|d(1)|$ will cause $\max |\beta(n)|$ to be large.

In our implementation, we choose to truncate the series at the point where $|\beta(n)|$ has passed a possible maximum value and $|\beta(n)|<\varepsilon$.

The same tests as with the asymptotic expansions suggest that for $x \leq X$, we obtain $\operatorname{Re} I_{i R}(x)$ to at least $P-6$ correct significant digits when $R \leq 50$, at least $P-11$ correct significant digits when $R \leq 100$, and at least $P-23$ correct significant digits when $R \leq 200$.

With $\operatorname{Im} I_{i R}(x)$, we depend on the accuracy of the PARI-routine besselk. The same tests show that this is a weak link in our overall accuracy. In fact, we will always call besselk with precision $P+19$, which ensures that $I_{i R}(x)$ will never lose accuracy due to besselk when $R \leq 200$.

Finally we tested these Bessel functions in our application (4-11). We compared values of

$$
\widehat{I}_{s-1 / 2}\left(2 \pi|m| v_{k}\right) \widehat{K}_{s-1 / 2}(2 \pi|m| y)
$$

computed with precisions $P$ and $P+19$ as they were generated by typical runs of the $G_{s}(z ; w)$-algorithm for nine different $R$-values between 1 and 200. The results are consistent with the individual $I$-Bessel and $K$-Bessel tests: the numbers of correct significant digits obtained are $P-5$ for $R \leq 50, P-11$ for $R \leq 100$, and $P-23$ for $R \leq 200$.

\section{NUMERICS}

Recall the truncated Fourier series (3-1) used to compute $F_{n}(z ; s)$ in Section 3. In order to estimate the error $R_{N}$ for $|n| \geq 1$ we use $(2-11)$ and $(2-1)$. By adding the absolute bounds for fixed $\Gamma, s$, and $n \neq 0$, we find for $0<y<1$ and $N \geq 4|n| /\left(c_{\Gamma}^{2} y^{2}\right)$ that

$$
\begin{aligned}
\left|R_{N}\right| & \leq O(1) \sum_{m \geq N} e^{4 \pi c_{\Gamma}^{-1} \sqrt{|n| m}-2 \pi m y} \\
& \leq O(1) e^{4 \pi c_{\Gamma}^{-1} \sqrt{|n| N}-2 \pi N y} \sum_{m \geq N} e^{-\pi y(m-N)} .
\end{aligned}
$$

The geometric series above is $O(1 / y)$, and so we have uniformly for all $0<y<1$ and all $N \geq 4|n| /\left(c_{\Gamma}^{2} y^{2}\right)$,

$$
R_{N}=O\left(y^{-1} e^{4 \pi c_{\Gamma}^{-1} \sqrt{|n| N}-2 \pi N y}\right) .
$$

The implied constant depends on $s, n$, and $\Gamma$. This sort of estimate may be used, combined with trial and error, to decide on an appropriate $M_{0}$-value. With $R \approx 14$ our numerics suggest that the implied constant in $(6-1)$ is roughly $e^{R \pi / 2}$ for $1 \leq|n| \leq 10$ and $y$ near 0.8 (recall here (4-3)), but with $R \approx 100$ it seems to be quite a bit larger.

We now turn to the tail $S_{N}$ of the Fourier series of $G_{s}^{r}(z ; w)$ truncated at $m=N$ (cf. $\left.(4-13)\right)$ :

$$
S_{N}=\sum_{|m| \geq N+1} f_{m}^{r} y^{1 / 2} \widehat{K}_{s-\frac{1}{2}}(2 \pi|m| y) e^{2 \pi i m x} .
$$

Let $\varepsilon>0$ be fixed. From the bound (4-12) together with $(2-1)$ it follows that

$$
\begin{aligned}
S_{N} & =O\left(\sum_{|m| \geq N+1} \frac{1}{|m|} e^{-2 \pi|m|\left(y-v_{r+1}-\varepsilon\right)}\right) \\
& <O\left(\frac{1}{N} e^{-2 \pi N\left(y-v_{r+1}-\varepsilon\right)}\right)
\end{aligned}
$$

holds for $y>v_{r+1}+2 \varepsilon$. Thus the accuracy in the $G_{s}^{r}(z ; w)$-computations will depend on $r$.

With some heuristics we can make the $S_{N}$-estimate more useful. Recall that

$$
f_{m}^{r}=f_{m}+\mu \sum_{k=0}^{r} \sum_{w^{\prime} \in H_{v_{k}}} v_{k}^{1 / 2} \widehat{I}_{s-\frac{1}{2}}\left(2 \pi|m| v_{k}\right) e^{-2 \pi i m u^{\prime}}
$$

with $f_{m}=-e^{-R \pi / 2} F_{-m}(w ; s)$, and that $H_{v_{k}}$ for $k=$ $0,1,2, \ldots$ was defined in such a way that all $w^{\prime} \in H_{v_{k}}$ have $\operatorname{Im} w^{\prime}=v_{k}$, and $v_{0}>v_{1}>v_{2}>\cdots$; cf. Section 4 . Assuming $N$ large enough we may write (2-5), for $|m| \geq$ $N+1$ and $\operatorname{Re} s>1$, as leading terms plus a remainder

$$
\begin{aligned}
& e^{-R \pi / 2} F_{-m}(w ; s) \\
& =\mu \sum_{k=0}^{r+1} \sum_{w^{\prime} \in H_{v_{k}}} v_{k}^{1 / 2} \widehat{I}_{s-\frac{1}{2}}\left(2 \pi|m| v_{k}\right) e^{-2 \pi i m u^{\prime}}+\xi,
\end{aligned}
$$


with

$$
\xi=O\left(v_{r+2}^{1 / 2} \widehat{I}_{s-\frac{1}{2}}\left(2 \pi|m| v_{r+2}\right)\right) .
$$

We now assume that (6-5) holds also for $\operatorname{Re} s=1 / 2$. For $s=1 / 2+i R$ we then obtain

$$
\begin{aligned}
\left|S_{N}\right| \leq & O(1) \cdot \mu \cdot \# H_{v_{r+1}} \\
& \times \sum_{m \geq N+1} \frac{1}{2 \pi m} e^{-2 \pi m y+\sqrt{\left(2 \pi m v_{r+1}\right)^{2}+R^{2}}} \\
< & O(1) \cdot \frac{\mu \cdot \# H_{v_{r+1}}}{2 \pi(N+1)} e^{-2 \pi N y+\sqrt{\left(2 \pi N v_{r+1}\right)^{2}+R^{2}}} .
\end{aligned}
$$

Here we have used (2-1) and estimated $I_{i R}(x)$ as $(2 \pi x)^{-1 / 2} e^{\sqrt{x^{2}+R^{2}}}$ (this seems to be suggested by [Olver 54] and [Erdélyi et al. 53, p. 87], but we have not found it in the literature). The tail estimate (6-6) has proven to be very useful for predicting a suitable truncation in our actual $G_{s}^{r}(z ; w)$-runs.

\section{RESULTS}

We present several statistical tests of $G_{s}(z ; w)$ and $F_{n}(z ; s)$ in [Avelin 10]. Here we show some results and a number of tests validating the $F_{n}(z ; s)$ and $G_{s}(z ; w)$ algorithms.

Let $\Gamma$ be the modular group $\operatorname{PSL}(2, \mathbb{Z})$. Then $\Gamma$ has one cusp, $i \infty$, and $\mathcal{R} \Gamma=\Gamma \mathcal{R}$ holds ( $\mathcal{R}$ was defined in Section 2). We take the fundamental polygon as $\mathcal{F}=$ $\{z \in \mathcal{H}:-1 / 2 \leq \operatorname{Re} z \leq 1 / 2,|z| \geq 1\}$. The lower righthand corner of $\mathcal{F}$ is

$$
\rho=\frac{1}{2}+i \frac{\sqrt{3}}{2} .
$$

If $s$ is a zero of the Riemann zeta function or a zero of the Dirichlet $L$-function $L\left(s,\left(\frac{-3}{\cdot}\right)\right)$, then $G_{s}(z ; \rho)$ is a pseudo cusp form; cf. [Hejhal 81, p. 99, 104-105].

Recall that (4-11) is valid for $y>v_{r+1}$ and that we need to be able to find a suitable $Y>v_{r+1}$ to proceed with (4-14). Moreover, from (6-3) we know that $r$ has to be adapted to the specific precision we choose. For the purposes of the present paper we can always stay above height $1 / 3$, i.e., we can keep $r$ so small that $v_{r}>1 / 3$. In this case, to find $v_{k}$ and $H_{v_{k}}$ for $k=0, \ldots, r$, we only have to consider the points $-1 /(w+a)$ for $a \in\{-1,0,1\}$, and (if $w \in \partial \mathcal{F}$ ) some of the $S$-translates of these points; every other point in $\Gamma w$ will necessarily have imaginary part less than or equal to $1 / 3$. (This is proved by studying the tessellation of $\mathcal{H}$ by the images $T \mathcal{F}, T \in \Gamma$.)

There are quite a few relations suitable for testing our algorithms for $F_{n}(z ; s)$ and $G_{s}(z ; w)$; see, for example, Lemma 2.1. Here we will exploit five of them (as natural "insurance devices"): (a) The computed Fourier coefficients are independent of the choice of $Y$.

(b) Coefficient relation: $\varphi_{m}^{n}(s)=\varphi_{n}^{m}(s)$.

(c) Automorphy.

(d) $F_{n}(z ; s)-F_{n}(z ; 1-s)=\varphi_{-n}^{0}(s) E(z ; 1-s) /(2 s-1)$.

(e) $F_{n}(w ; s)$ is a Fourier coefficient of $G_{s}(z ; w)$; cf. $(2-4)$.

With $\operatorname{Re} s=1 / 2$, (d) becomes $F_{n}(z ; s)-F_{n}(z ; \bar{s})=$ $\varphi_{-n}^{0}(s) E(z ; \bar{s}) /(2 i R)$, and using $E(z ; \bar{s})=\overline{E(z ; s)}$ together with $F_{n}(z ; \bar{s})=\overline{F_{-n}(z ; s)}$ and $\varphi_{-n}^{0}(s)=\varphi_{n}^{0}(s)$, cf. Lemma 2.1 and the remark following $(2-5)$, we obtain

$$
F_{n}(z ; s)-\overline{F_{-n}(z ; s)}=\frac{\varphi_{n}^{0}(s) \overline{E(z ; s)}}{2 i R} .
$$

If $\mathcal{R} w=T w$ for some $T \in \Gamma$, then $G_{s}(z ; w)$ is even and $F_{n}(w ; s)=F_{-n}(w ; s)$; cf. Lemma 2.1(d),(e). Then $(7-1)$ becomes

$$
\operatorname{Im} F_{n}(w ; s)=-\frac{\varphi_{n}^{0}(s) \overline{E(w ; s)}}{4 R} .
$$

For example, this means that if $\zeta(s)=0$, then $F_{n}(\rho ; s) \in \mathbb{R}$ and $G_{s}(z ; \rho) \in \mathbb{R} ;$ cf. [Hejhal 81, p. 105]. The coefficients $\varphi_{n}^{0}(s)$ of the Eisenstein series on $\operatorname{PSL}(2, \mathbb{Z})$ may be computed via [Hejhal 83, p. 76$]$.

The testing of output data was built up as follows: Tests (a) and (b) for $F_{n}(z ; s)$-coefficients, (c) and (d) for $F_{n}(z ; s)$-values, and then finally, (a) and (e) as tests for $G_{s}(z ; w)$-coefficients.

In the following few pages we will discuss as an example the first Riemann zero, i.e., $s=1 / 2+i R$ with $R=14.134725 \ldots$, and working precision $P=38$. A number of tests were performed with $R \leq 98.831194 \ldots$, $1 \leq|n| \leq 10$, and $P \leq 57$. (Note that $R=98.831194 \ldots$ also corresponds to a Riemann zero.) The overall picture was the same as described below in all cases, as well as the explicit " $P-m$ "-type results.

The truncation of the Fourier series for $F_{n}(z ; s)$ (cf. $(3-1)$ ) for our example may be decided using (6-1) (more precisely, its counterpart for (3-4)); cf. Section 6 . We choose $M_{0}$ as the smallest positive integer such that $K_{s-1 / 2}(2 \pi|m| Y)$ decays exponentially as $|m| \geq M_{0}$ increases, and

$$
e^{4 \pi \sqrt{\left|M_{0} n\right|}-2 \pi\left|M_{0}\right| Y-R \pi / 2}<10^{-P} .
$$

(We used $Y=0.85$ or $Y=0.86$ and $M_{0}$-values $30,31,38$, $44,50,56,62,68,74,79$ for $|n|=1, \ldots, 10$.)

Examples of the tests (a) and (b) are presented in Tables 1 and 2 . 


\begin{tabular}{|r|r|r|c|c|}
\hline$n$ & $m$ & $e^{-R \pi} \varphi_{m}^{n}(s)$ & $(\mathrm{b})$ & $(\mathrm{a})$ \\
\hline 1 & 0 & $0.6363033313251248455-0.34893959844819293002 i$ & $3 \mathrm{E}-37$ & $3 \mathrm{E}-38$ \\
1 & 1 & $0.1522935306982800404+0.14984028066408378613 i$ & - & $8 \mathrm{E}-37$ \\
1 & -1 & $-0.2934371142810432546-0.00931466242781154964 i$ & - & $5 \mathrm{E}-37$ \\
1 & 2 & $-0.1878701251370421943+0.01735060114265149564 i$ & $1 \mathrm{E}-36$ & $5 \mathrm{E}-38$ \\
1 & -2 & $-0.08209516517515990832+0.01735060114265149564 i$ & $1 \mathrm{E}-36$ & $3 \mathrm{E}-37$ \\
1 & 3 & $0.1133719416917023190+0.01833040629870669329 i$ & $3 \mathrm{E}-35$ & $1 \mathrm{E}-36$ \\
1 & -3 & $-0.2288270016347127611+0.01833040629870669329 i$ & $2 \mathrm{E}-35$ & $4 \mathrm{E}-37$ \\
1 & 4 & $-0.3454996504239681168-0.02300463656390817151 i$ & $1 \mathrm{E}-33$ & $2 \mathrm{E}-36$ \\
1 & -4 & $0.09351644845644996581-0.02300463656390817151 i$ & $4 \mathrm{E}-33$ & $3 \mathrm{E}-36$ \\
1 & 5 & $\dagger-0.3262149786472086677+0.01353122930133739777 i$ & $1 \mathrm{E}-30$ & $7 \mathrm{E}-35$ \\
1 & -5 & $\ddagger 0.4458721693009334992+0.01353122930133739777 i$ & $7 \mathrm{E}-31$ & $2 \mathrm{E}-35$ \\
1 & 6 & $-0.03298516237881328331-0.03414440093094493948 i$ & $9 \mathrm{E}-28$ & $5 \mathrm{E}-32$ \\
1 & -6 & $0.4909254746752517213-0.03414440093094493948 i$ & $1 \mathrm{E}-28$ & $7 \mathrm{E}-33$ \\
1 & 7 & $-0.1320743969307616161+0.01338159684868249107 i$ & $1 \mathrm{E}-25$ & $4 \mathrm{E}-31$ \\
1 & -7 & $1.34103063072489491+0.01338159684868249107 i$ & $3 \mathrm{E}-26$ & $3 \mathrm{E}-31$ \\
1 & 8 & $0.09163133101247650488+0.02550057854801231049 i$ & $9 \mathrm{E}-22$ & $7 \mathrm{E}-28$ \\
1 & -8 & $3.93755307502619889+0.02550057854801231049 i$ & $3 \mathrm{E}-23$ & $2 \mathrm{E}-29$ \\
1 & 9 & $-0.01763591827357110023-0.02675790570653457503 i$ & $2 \mathrm{E}-18$ & $2 \mathrm{E}-25$ \\
1 & -9 & $14.9294661703475675-0.02675790570653457503 i$ & $8 \mathrm{E}-21$ & $4 \mathrm{E}-28$ \\
1 & 10 & $-0.0729070547389021093-0.02520488148623370828 i$ & $2 \mathrm{E}-16$ & $4 \mathrm{E}-23$ \\
1 & -10 & $55.488453466882473-0.02520488148623370828 i$ & $7 \mathrm{E}-19$ & $1 \mathrm{E}-26$ \\
\hline
\end{tabular}

TABLE 1. Fourier coefficients computed with the $F_{n}(z ; s)$-algorithm and estimated relative errors, for $s=1 / 2+$ $i 14.134725 \ldots, n=1$, and precision $P=38$. Concerning the factor $e^{-R \pi}$, see $(3-4)$.

\begin{tabular}{|r|r|r|c|c|}
\hline$n$ & $m$ & $e^{-R \pi} \varphi_{m}^{n}(s)$ & $(\mathrm{b})$ & $(\mathrm{a})$ \\
\hline 5 & 0 & $-0.9243454980888676213+0.50689778137546680617 i$ & $1 \mathrm{E}-31$ & $1 \mathrm{E}-31$ \\
5 & 1 & $\dagger-0.3262149786472086677+0.01353122930133739777 i$ & $1 \mathrm{E}-31$ & $1 \mathrm{E}-30$ \\
5 & -1 & $\ddagger 0.4458721693009334992+0.01353122930133739777 i$ & $1 \mathrm{E}-31$ & $1 \mathrm{E}-30$ \\
5 & 2 & $-0.07290705473890210931-0.02520488148623370828 \mathrm{i}$ & $6 \mathrm{E}-31$ & $1 \mathrm{E}-30$ \\
5 & -2 & $55.48845346688247383-0.02520488148623370828 \mathrm{i}$ & $7 \mathrm{E}-34$ & $2 \mathrm{E}-34$ \\
5 & 3 & $-0.2712539339231707466-0.02662822541737072424 i$ & $1 \mathrm{E}-31$ & $1 \mathrm{E}-30$ \\
5 & -3 & $43177.92568424207870-0.02662822541737072424 i$ & $1 \mathrm{E}-36$ & $8 \mathrm{E}-36$ \\
5 & 4 & $0.20663575107822840348+0.03341838899182806006 i$ & $2 \mathrm{E}-29$ & $7 \mathrm{E}-30$ \\
5 & -4 & $21916908.345622582336+0.03341838899182806006 i$ & $1 \mathrm{E}-37$ & $2 \mathrm{E}-37$ \\
5 & 5 & $0.3825060743644429903+0.13949838894145263907 i$ & - & $2 \mathrm{E}-28$ \\
5 & -5 & $6964160617.220798386-0.01965655415044269670 i$ & - & $5 \mathrm{E}-38$ \\
5 & 6 & $0.11208007498730239125+0.04960090845310034638 i$ & $6 \mathrm{E}-25$ & $1 \mathrm{E}-25$ \\
5 & -6 & $1478576045205.8738879+0.04960090845310034638 i$ & $3 \mathrm{E}-37$ & $4 \mathrm{E}-38$ \\
5 & 7 & $0.19127124173693323782-0.01943918599099660566 i$ & $3 \mathrm{E}-22$ & $1 \mathrm{E}-23$ \\
5 & -7 & $223832024724515.27314-0.01943918599099660566 i$ & $1 \mathrm{E}-36$ & $4 \mathrm{E}-38$ \\
5 & 8 & $-0.19347852196781997140-0.03704419546323692509 i$ & $2 \mathrm{E}-19$ & $1 \mathrm{E}-21$ \\
5 & -8 & $25471921532847243.610-0.03704419546323692509 i$ & $6 \mathrm{E}-36$ & $1 \mathrm{E}-37$ \\
5 & 9 & $0.18140776376700638375+0.03887069022035942775 i$ & $4 \mathrm{E}-16$ & $1 \mathrm{E}-19$ \\
5 & -9 & $2271845180732047467.2+0.03887069022035942770 i$ & $1 \mathrm{E}-34$ & $7 \mathrm{E}-38$ \\
5 & 10 & $-0.14834699089805970912+0.03661464208138688354 i$ & $6 \mathrm{E}-13$ & $5 \mathrm{E}-17$ \\
5 & -10 & $164141060422096157650+0.03661464208138687450 i$ & $1 \mathrm{E}-34$ & $1 \mathrm{E}-37$ \\
\hline
\end{tabular}

TABLE 2. Fourier coefficients computed with the $F_{n}(z ; s)$-algorithm and estimated relative errors, for $s=1 / 2+$ $i 14.134725 \ldots, n=5$, and precision $P=38$. Concerning the factor $e^{-R \pi}$; see $(3-4)$. 


\begin{tabular}{|c|c|c|c|c|c|}
\hline$n$ & $z$ & $z^{*}$ & $(\mathrm{c})$ & $(\mathrm{d}) z$ & $(\mathrm{~d}) z^{*}$ \\
\hline 1 & $\rho$ & - & - & $8 \mathrm{E}-38$ & - \\
1 & $0.49+\sqrt{1-0.49^{2}} \mathrm{i}$ & - & - & $8 \mathrm{E}-38$ & - \\
1 & $0.4+0.8 \mathrm{i}$ & $0.5+1.0 \mathrm{i}$ & $3 \mathrm{E}-34$ & $3 \mathrm{E}-35$ & $8 \mathrm{E}-39$ \\
1 & $0.2+0.85 \mathrm{i}$ & $-0.2622950820+1.114754098 \mathrm{i}$ & $4 \mathrm{E}-38$ & $1 \mathrm{E}-36$ & $9 \mathrm{E}-37$ \\
1 & $0+0.87 \mathrm{i}$ & $0+1.149425287 \mathrm{i}$ & $8 \mathrm{E}-38$ & $9 \mathrm{E}-39$ & $1 \mathrm{E}-37$ \\
1 & $0+0.9 \mathrm{i}$ & $0+1.11111111 \mathrm{i}$ & $4 \mathrm{E}-38$ & $3 \mathrm{E}-38$ & $9 \mathrm{E}-38$ \\
1 & $-0.4+0.9 \mathrm{i}$ & $0.4123711341+0.927835051 \mathrm{i}$ & $9 \mathrm{E}-37$ & $1 \mathrm{E}-36$ & $2 \mathrm{E}-36$ \\
\hline 5 & $\rho$ & - & - & $1 \mathrm{E}-35$ & - \\
5 & $0.49+\sqrt{1-0.49^{2}} \mathrm{i}$ & - & - & $1 \mathrm{E}-35$ & - \\
5 & $0.4+0.8 \mathrm{i}$ & $0.5+1.0 \mathrm{i}$ & $5 \mathrm{E}-30$ & $4 \mathrm{E}-30$ & $1 \mathrm{E}-37$ \\
5 & $0.2+0.85 \mathrm{i}$ & $-0.2622950820+1.114754098 \mathrm{i}$ & $7 \mathrm{E}-37$ & $2 \mathrm{E}-37$ & $1 \mathrm{E}-37$ \\
5 & $0+0.87 \mathrm{i}$ & $0+1.149425287 \mathrm{i}$ & $3 \mathrm{E}-38$ & $1 \mathrm{E}-38$ & $4 \mathrm{E}-39$ \\
5 & $0+0.9 \mathrm{i}$ & $0+1.11111111 \mathrm{i}$ & $3 \mathrm{E}-37$ & $1 \mathrm{E}-38$ & $1 \mathrm{E}-38$ \\
5 & $-0.4+0.9 \mathrm{i}$ & $0.4123711341+0.927835051 \mathrm{i}$ & $1 \mathrm{E}-35$ & $2 \mathrm{E}-36$ & $3 \mathrm{E}-36$ \\
\hline 10 & $\rho$ & - & - & $2 \mathrm{E}-31$ & - \\
10 & $0.49+\sqrt{1-0.49^{2}} \mathrm{i}$ & - & - & $1 \mathrm{E}-31$ & - \\
10 & $0.4+0.8 \mathrm{i}$ & $0.5+1.0 \mathrm{i}$ & $1 \mathrm{E}-22$ & $1 \mathrm{E}-23$ & $1 \mathrm{E}-34$ \\
10 & $0.2+0.85 \mathrm{i}$ & $-0.2622950820+1.114754098 \mathrm{i}$ & $5 \mathrm{E}-36$ & $5 \mathrm{E}-37$ & $9 \mathrm{E}-38$ \\
10 & $0+0.87 \mathrm{i}$ & $0+1.149425287 \mathrm{i}$ & $1 \mathrm{E}-37$ & $2 \mathrm{E}-38$ & $1 \mathrm{E}-38$ \\
10 & $0+0.9 \mathrm{i}$ & $0+1.11111111 \mathrm{i}$ & $3 \mathrm{E}-37$ & $1 \mathrm{E}-37$ & $9 \mathrm{E}-38$ \\
10 & $-0.4+0.9 \mathrm{i}$ & $0.4123711341+0.927835051 \mathrm{i}$ & $2 \mathrm{E}-33$ & $5 \mathrm{E}-33$ & $6 \mathrm{E}-33$ \\
\hline
\end{tabular}

TABLE 3. Estimated accuracy of $F_{n}(z ; s)$-values for $s=1 / 2+i 14.134725 \ldots$ with precision $P=38$.

\begin{tabular}{|c|r|c|c|}
\hline$n$ & \multicolumn{1}{|c|}{$f_{n}$} & $(\mathrm{a})$ & $(\mathrm{e})$ \\
\hline 1 & 0.1235571424212640662462323300904779518 & $1 \mathrm{E}-36$ & $1 \mathrm{E}-36$ \\
2 & 0.5596998544549825388708785270788401870 & $7 \mathrm{E}-36$ & $7 \mathrm{E}-36$ \\
3 & 0.9842653921361874364144161469066487430 & $4 \mathrm{E}-35$ & $4 \mathrm{E}-35$ \\
4 & -21.2118402111191571768351437598366002122 & $5 \mathrm{E}-34$ & $5 \mathrm{E}-34$ \\
5 & 1495.2634314255648996494000184233186794846 & $6 \mathrm{E}-32$ & $5 \mathrm{E}-32$ \\
6 & -159357.5971037973419533555408670690353972527 & $1 \mathrm{E}-29$ & $1 \mathrm{E}-29$ \\
7 & 21220217.7779904655339084525264672439636772917 & $6 \mathrm{E}-27$ & $5 \mathrm{E}-27$ \\
8 & -3232778831.6108074346436944272250611603987909810 & $3 \mathrm{E}-25$ & $2 \mathrm{E}-25$ \\
9 & 538008642234.8093221305756252886064623889331697296 & $9 \mathrm{E}-24$ & $8 \mathrm{E}-24$ \\
10 & -95218023964901.9252028793820595301380072713199342494 & $4 \mathrm{E}-22$ & $4 \mathrm{E}-22$ \\
\hline
\end{tabular}

TABLE 4. Values of Fourier coefficients $f_{n}$ of $G_{s}(z ; w)$ for $w=\rho, s=1 / 2+i 14.134725 \ldots$, and relative error estimates for the corresponding $f_{n}^{r}$. Precision $P=38$. Cf. (4-2) and (4-3) à propos $f_{n}$.

These tables include values of $e^{-R \pi} \varphi_{m}^{n}(s)$ computed using $Y=0.86$ for $n=1,5$ and $|m| \leq 10$. For each $\varphi_{m}^{n}(s)$, errors estimated with the tests (a) and (b) are presented. These are computed as the relative difference between $\varphi_{m}^{n}(s)$ and the appropriate $\varphi_{ \pm n}^{|m|}(s)$ (where we use Lemma 2.1(f) and the fact that computations of $F_{-m}(z ; s)$ are entirely similar to computations of $\left.F_{m}(z ; s)\right)$ for (b); and between $\varphi_{m}^{n}(s)$ and the same $\varphi_{m}^{n}(s)$ computed with a different $Y$-value (namely, $Y=0.85$ ) for (a).

Coefficients corresponding to each other by the relations in Lemma 2.1(f) and (g) are marked with $\dagger$ and $\ddagger$.

For $\operatorname{Re} s>1$ there is a formula for $\varphi_{m}^{n}(s)$ involving Kloosterman sums and $J$ - or $I$-Bessel functions for $n m$ positive or negative, respectively; cf. [Hejhal 83, p. 62] or [Niebur 73, pp. 134, 135]. Both these Bessel factors grow with $\sqrt{|n m|}$, but the $I$-Bessel factor grows significantly faster than the $J$-Bessel counterpart; see, for example, [Watson 44, pp. 202-203]. The effect is that $\varphi_{m}^{n}(s)$ for (say) $n, m \geq 1$ is several orders of magnitude smaller than $\varphi_{-m}^{n}(s)$ already for moderate values of $m$. We expect the same behavior for $\operatorname{Re} s<1$; it seems likely that a rigorous statement along these lines should be possible to prove using the techniques involved in the BruggemanKuznetsov formula; see, for example, [Iwaniec 02, Chapter 9, especially p. 126]. We then also expect a loss of accuracy in the smaller of the two "twin coefficients" $\varphi_{ \pm m}^{n}(s)$ due to cancellation. The number of significant digits lost should be about the difference in size between the two "twin coefficients." See, for example, $m= \pm 10$ in Table 2. 


\begin{tabular}{|c|r|r|c|}
\hline$n$ & $f_{n}$ & $(\mathrm{a})$ & $(\mathrm{e})$ \\
\hline 1 & -0.125612706297120441197377827995215897 & $2 \mathrm{E}-36$ & $2 \mathrm{E}-36$ \\
& $+0.104016854037173288045865304745288921 * \mathrm{I}$ & & \\
\hline 2 & 0.062850063635789652855553590939931039 & $3 \mathrm{E}-37$ & $3 \mathrm{E}-37$ \\
& $+0.165676016580456187957735506454237166 * \mathrm{I}$ & & \\
\hline 3 & 12.838493976890135235372284633892315642 & $1 \mathrm{E}-36$ & $1 \mathrm{E}-36$ \\
& $-9.253727669489156067384615977985770957 * \mathrm{I}$ & & \\
\hline 4 & -2200.661437827674338619554451320423209849 & $6 \mathrm{E}-36$ & $6 \mathrm{E}-36$ \\
& $-6780.169555693669003112475390886578135093 * \mathrm{I}$ & & \\
\hline 5 & -5829928.467676691382258185366552113255828156 & $5 \mathrm{E}-34$ & $5 \mathrm{E}-34$ \\
& $+104.197776966256971515268573968829457292 * \mathrm{I}$ & & \\
\hline 6 & $+5958473213.369427376325439802783488677683140604 * \mathrm{I}$ & & \\
\hline 7 & $+4610951345790.268048863674111374708151972068419770 * \mathrm{I}$ & & \\
\hline 8 & -6739138277785946.432686483186214146720899151324876617 & $8 \mathrm{E}-30$ & $7 \mathrm{E}-29$ \\
& -4898292916234117542.591509156906049227562052640091720612 & $4 \mathrm{E}-27$ & $3 \mathrm{E}-27$ \\
\hline 9 & $-15075395471564365969.056335541002096201826922782201807132 * \mathrm{I}$ & & \\
\hline 10 & -24326462439647508260569.5995007293335530184865743622561718 & $1 \mathrm{E}-25$ & $1 \mathrm{E}-25$ \\
& $-905138289572.893220513362613203976239879656387896 * \mathrm{I}$ & & \\
\hline
\end{tabular}

TABLE 5. Values of Fourier coefficients $f_{n}$ of $G_{s}(z ; w)$ for $w=0.2+1.2 i, s=1 / 2+i 14.134725 \ldots$, and relative error estimates for the corresponding $f_{n}^{r}$. Precision $P=38$. Cf. Cf. (4-2) and (4-3) à propos $f_{n}$.

\begin{tabular}{|c|r|c|}
\hline$n$ & {$[$ Hejhal 92] } & $f_{n} / f_{1}$ \\
\hline 2 & 4.5300 & 4.5298866863293426919271769474038512688 \\
3 & 7.967 & 7.9660744239322606909165900956187083269 \\
4 & $-1.717 \mathrm{E} 2$ & -171.6763579623594445335087109705928922 \\
5 & $1.211 \mathrm{E} 4$ & 12101.79680529930652299605780712641026514 \\
6 & $-1.291 \mathrm{E} 6$ & -1289748.1600899508310111788873067778738 \\
7 & $1.719 \mathrm{E} 8$ & 171744161.13995920897912219514475210879 \\
8 & $-2.62 \mathrm{E} 10$ & -26164240838.370581190895242569506670557 \\
9 & $4.37 \mathrm{E} 12$ & 4354330568770.2481897011772183388222484 \\
10 & $-7.75 \mathrm{E} 14$ & -770639576951846.00017592241755471420797 \\
\hline
\end{tabular}

TABLE 6. A comparison between Fourier coefficients of $G_{s}(z ; \rho)$ with $s=1 / 2+i 14.134725 \ldots$ from [Hejhal 92, p. 56] and the same coefficients computed with the $G_{s}(z ; w)$-algorithm. Here the coefficients are normalized by $f_{1}$.

Usually with these types of algorithms the leading Fourier coefficients are produced with high accuracy and the accuracy decreases as the significance of the terms in the Fourier expansion decreases. In $(2-6)$, the leading terms are those $\varphi_{m}^{n}(s) y^{1 / 2} K_{s-1 / 2}(2 \pi|m| y) e^{2 \pi i m x}$ with $|m| \approx|n| /\left(c_{\Gamma}^{2} y^{2}\right)$; cf. (2-11) and (2-1). Therefore it is natural to expect less accuracy for coefficients with $|\mathrm{m}|$ significantly smaller than $|n| /\left(c_{\Gamma}^{2} y^{2}\right)$ than for coefficients with $|m|$ near $|n| /\left(c_{\Gamma}^{2} y^{2}\right)$. This effect is visible for $n=5$ in Table 2 (where $|n| /\left(c_{\Gamma}^{2} y^{2}\right) \approx 6.7$ ) in comparing, for example, $m=-1$ with $m=-7$.

When Fourier coefficients $\varphi_{m}^{n}(s)$ of $F_{n}(z ; s)$ are computed for $-M_{0} \leq m \leq M_{0}$, we found, for this particular example, that $(3-1)$ will produce $F_{n}(z ; s)$-values with good result when truncated at $M=M_{0}-2$ if $y \geq 0.85$.
The tests $(\mathrm{c})$ and $(\mathrm{d})$ for $F_{n}(z ; s)$-values are consistent. They both indicate that the accuracy is, in general, decreasing with increasing $|n|$ for $z$ fixed, and that the poorest (relative) accuracy is for $|z|$ near 1 . (It seems that $\left|F_{n}(z ; s)\right|$ is particularly small for $|z|=1$. We hope to discuss the magnitude of $F_{n}(z ; s)$ in a forthcoming paper.)

Some sample data are presented in Table 3. All error estimates are relative. As in Section 3, $z^{*}$ denotes the image of $z$ in $\mathcal{F}$. Note that the value $z=0.4+0.8 i$ has imaginary part smaller than is optimal for our $M$ value; that is, the tail $\left|R_{M}\right|$ is greater than $10^{-38}$, which is reflected in the accuracy of $F_{n}(z ; s)$. The relative difference between $F_{n}(z ; s)$ and $F_{n}\left(z^{*} ; s\right)$ is in the column marked (c); the difference between the left-hand side and 

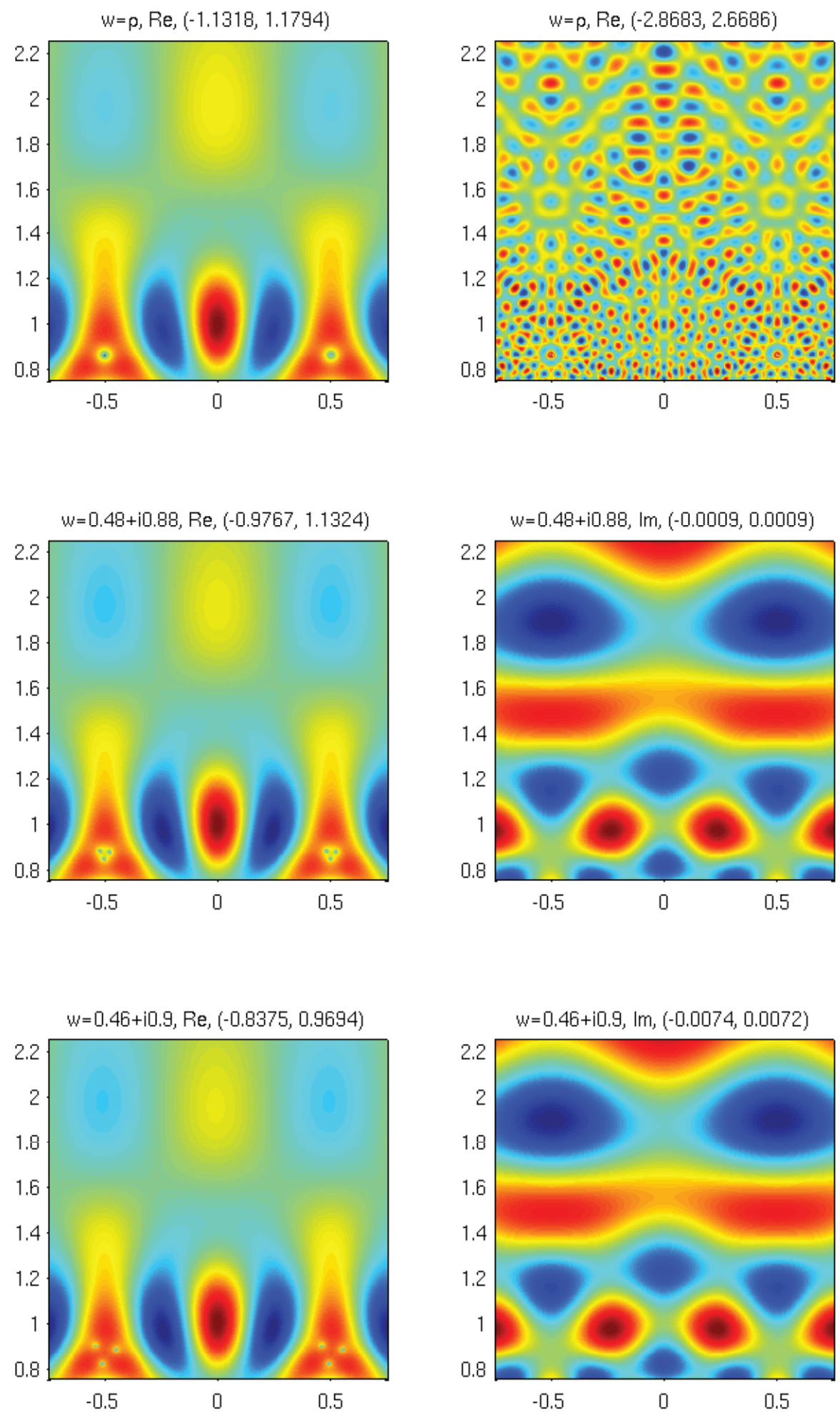

FIGURE 1. Pictures of $G_{s}(z ; w)$. The first two pictures show pseudo cusp forms with $w=\rho$; and $s=1 / 2+i 14.13 \ldots$ and $s=1 / 2+i 98.83 \ldots$, on the left- and right-hand sides, respectively. The next two rows show the real and imaginary parts of $G_{s}(z ; w)$ for perturbations of the location of the pole and fixed $s=1 / 2+i 14.13 \ldots$ These are not pseudo cusp forms because $E(w ; s) \neq 0$. Minimum and maximum values are given in parentheses. 
the right-hand side of $(7-1)$ divided by $\left|F_{n}(z ; s)\right|$ is in the column marked (d) $z$, and correspondingly for (7-1) applied to $z^{*}$.

At this point we are confident that our code produces $F_{n}(z ; s)$-values to at least $P-7$ places for $1 \leq|n| \leq 10$, $|m| \leq 10$. Thus test (e) will reveal the accuracy of our algorithm for $G_{s}(z ; w)$-coefficients. Tests were performed for several $w$-values, and Tables 4 and 5 show our results for $w=\rho$ and $w=0.2+1.2 i$ respectively. These tables include values of $f_{n}$ computed as Fourier coefficients of $G_{s}(z ; w)$ via $G_{s}^{r}(z ; w)$; cf. (4-2), (4-11), with $Y=0.86$, $M_{0}=24, s=1 / 2+i 14.134725 \ldots, P=38$. The third column is the relative error in $f_{n}^{r}$ estimated with test (a) and $Y=0.85,0.86$. The fourth column is the relative difference between $f_{n}^{r}$ and

$$
\begin{aligned}
& -e^{-R \pi / 2} F_{-n}(z ; s) \\
& +\mu \sum_{k=0}^{r} \sum_{w^{\prime} \in H_{v_{k}}} v_{k}^{1 / 2} \widehat{I}_{s-1 / 2}\left(2 \pi|m| v_{k}\right) e^{-2 \pi i m u^{\prime}}
\end{aligned}
$$

where $F_{-n}(z ; s)$ is computed with our $F_{n}(z ; s)$-algorithm using precision $P+19$ (which ensures at least $P$ correct significant digits); cf. (6-4) and test (e).

It appears that the $G_{s}(z ; w)$-algorithm produces $f_{n^{-}}^{r^{-}}$ values with at least $P-16$ correct significant digits for $1 \leq|n| \leq 10$. The accuracy in the corresponding $f_{n}$ depends on the size and accuracy of the computed value of $\mu \sum_{k=0}^{r} \sum_{w^{\prime} \in H_{v_{k}}} v_{k}^{1 / 2} \widehat{I}_{s-1 / 2}\left(2 \pi|m| v_{k}\right) e^{-2 \pi i m u^{\prime}}$. In Table 4 , all $f_{n}$ actually have about 36 significant digits. (Also recall (7-2) and the paragraph following it.)

In [Hejhal 92], the author computed $G_{s}(z ; \rho)$ with $s=1 / 2+i 14.1347 \ldots$ by diminishing the sharpness of his cusp form algorithm enough to pick up some pseudo cusp forms as well. Table 6 repeats some of his findings and compares them to our data. (Note that his computation yielded a slightly perturbed 14.134739 instead of 14.134725, while we have used 14.134725.)

We conclude by showing some pictures of $G_{s}(x+i y ; w)$ on $\operatorname{PSL}(2, \mathbb{Z})$ with $-0.75<x<0.75,0.75<y<2.25$, and $w=\rho, 0.48+0.88 i, 0.46+0.9 i$. (Recall that $G_{s}(z ; \rho) \in \mathbb{R}$ by Lemma 2.1 ; cf. the discussion near (7-2).) We have computed values at $500 \times 500$ points and given them colors ranging through blue, green, yellow, and red as the values pass from their minimum to their maximum, except for points very near the singularities, which have been given the value 0 and therefore are green; cf. Figure 1.

\section{ACKNOWLEDGMENTS}

The author is most grateful to Dennis Hejhal, Fredrik Strömberg, and Andreas Strömbergsson for helpful discussions on this work.

\section{REFERENCES}

[Abramowitz and Stegun 64] M. Abramowitz and I. A. Stegun (editors). Handbook of Mathematical Functions. New York: Dover, 1964.

[Avelin 07] H. Avelin. "Deformation of $\Gamma_{0}(5)$-Cusp Forms." Math. Comp. 76 (2007), 361-384.

[Avelin 10] H. Avelin. "Numerical Computations of the Green's Function and Its Fourier Coefficients on $\operatorname{PSL}(2, \mathbb{Z})$." Exp. Math. 19:3 (2010), 335-343.

[Barnard 03] A. Barnard. "The Singular Theta Correspondence, Lorentzian Lattices and Borcherds-Kac-Moody Algebras." PhD thesis, U.C. Berkeley, 2003.

[Bender and Orszag 99] C. M. Bender and S. A. Orszag, Advanced Mathematical Methods for Scientists and Engineers, Asymptotic Methods and Perturbation Theory. New York: Springer, 1999.

[Borcherds 98] R. E. Borcherds. "Automorphic Forms with Singularities on Grassmannians." Invent. math. 132 (1998), 491-562.

[Boyd 05] J. P. Boyd. "Hyperasymptotics and the Linear Boundary Layer Problem: Why Asymptotic Series Diverge." SIAM Review 47:3 (2005), 553-575.

[Brunier 02] J. H. Bruinier. Borhcerds Products on $\mathrm{O}(2, l)$ and Chern Classes of Heegner Divisors, Lecture Notes in Math. 1780. New York: Springer, 2002.

[Conrey 03] J. B. Conrey. "The Riemann Hypothesis." Notices of the AMS 50:3 (2003), 341-353.

[Erdélyi et al. 53] A. Erdélyi, W. Magnus, F. Oberhettinger, and F. G. Tricomi, Higher Transcendental Functions, vol. 2. New York: McGraw-Hill, 1953.

[Fay 77] J. D. Fay. "Fourier Coefficients of the Resolvent for a Fuchsian Group." J. Reine Angew. Math. 293/294 (1977), 143-203.

[Gil et al. 02] A. Gil, J. Segura, and N. M. Temme. "Evaluation of the Modified Bessel Function of the Third Kind of Imaginary Order." Journal of Computational Physics 715 (2002), 398-411.

[Gil et al. 03] A. Gil, J. Segura, and N. M. Temme. "Computation of the Modified Bessel Function of the Third Kind of Imaginary Orders: Uniform Airy-Type Asypmtotic Expansion." Journal of Computational and Applied Mathematics 153 (2003), 225-234.

[Gil et al. 04a] A. Gil, J. Segura, and N. M. Temme. "Algorithm 831: Modified Bessel Functions of Imaginary Order and Positive Argument." ACM Trans. Math. Softw. 30 (2004), 159-164. 
[Gil et al. 04b] A. Gil, J. Segura, and N. M. Temme. "Computating Solutions of the Modified Bessel Differential Equation for Imaginary Orders and Positive Arguments." ACM Trans. Math. Softw. 30 (2004), 145-158.

[Hejhal 76] D. A. Hejhal. The Selberg Trace Formula for $\operatorname{PSL}(2, \mathbb{R})$, vol. 1, Lecture Notes in Math. 548. New York: Springer, 1976.

[Hejhal 81] D. A. Hejhal. "Some Observations Concerning Eigenvalues of the Laplacian and Dirichlet $L$-Series." In Recent Progress in Analytic Number Theory, vol. 2, pp. 95110. New York: Academic Press, 1981.

[Hejhal 83] D. A. Hejhal. The Selberg Trace Formula for $\operatorname{PSL}(2, \mathbb{R})$, vol. 2, Lecture Notes in Math. 1001. Berlin: Springer, 1983.

[Hejhal 92] D. A. Hejhal. Eigenvalues of the Laplacian for Hecke Triangle Groups, Mem. Amer. Math. Soc. 469, 1992.

[Hejhal 99] D. A. Hejhal. "On Eigenfunctions of the Laplacian for Hecke Triangle Groups." In Emerging Applications of Number Theory, edited by D. Hejhal, J. Friedman, M. Gutzwiller, and A. Odlyzko, pp. 291-315. New York: Springer, 1999.

[Iwaniec 97] H. Iwaniec. Topics in Classical Automorphic Forms. Providence: American Mathematical Society, 1997.

[Iwaniec 02] H. Iwaniec. Spectral Methods of Automorphic Forms, 2nd ed. Providence: American Mathematical Society, 2002.
[Marklof and Strömbergsson 03] J. Marklof and A. Strömbergsson. "Equidistribution of Kronecker sequences along Closed Horocycles. Geom. Funct. Anal. 13 (2003), 12391280.

[Niebur 73] D. Niebur. "A Class of Nonanalytic Automorphic Functions." Nagoya Math. J. 52 (1973), 133-145.

[Odlyzko 09] A. M. Odlyzko. "Correspondence about the Origins of the Hilbert-Pólya Conjecture." Available online (http://www.dtc.umn.edu/ odlyzko/polya/index .html), accessed 2009.

[Olver 54] F. W. J. Olver. "The Asymptotic Expansion of Bessel Functions of Large Order." Philos. Trans. Roy. Soc. London Ser. A 247 (1954), 328-368.

[Olver 74] F. W. J. Olver. Asymptotics and Special Functions. New York: Academic Press 1974.

[Selberg 56] A. Selberg. "Harmonic Analysis and Discontinuous Groups in Weakly Symmetric Riemannian Spaces with Applications to Dirichlet Series." J. Indian Math. Soc. B 20 (1956), 47-87.

[Shimizu 63] H. Shimizu. "On Discontinuous Subgroups Operating on the Oroduct of the Upper Half Planes." Ann. of Math. 77 (1963), 33-71.

[Strömbergsson 04] A. Strömbergsson. "On the Uniform Equidistribution of Long Closed Horocycles." Duke Math. J. $123: 3$ (2004), 507-543.

[Watson 44] G. N. Watson. A Treatise on the Theory of Bessel Functions, 2nd ed. Cambridge, UK: Cambridge University Press, 1944.

Helen Avelin, Department of Mathematics, Uppsala University, S-751 06 Uppsala, Sweden (helen.avelin@gmail.com)

Received June 1, 2007; accepted in revised form August 18, 2009. 